Bull. Soc. math. France

129 (4), 2001, p. 505-542

\title{
A MEAN-VALUE LEMMA AND APPLICATIONS
}

\author{
By Alessandro Savo
}

\begin{abstract}
We control the gap between the mean value of a function on a submanifold (or a point), and its mean value on any tube around the submanifold (in fact, we give the exact value of the second derivative of the gap). We apply this formula to obtain comparison theorems between eigenvalues of the Laplace-Beltrami operator, and then to compute the first three terms of the asymptotic time-expansion of a heat diffusion process on convex polyhedrons in euclidean spaces of arbitrary dimension. We also write explicit bounds for the remainder term of the above expansion, which hold for all values of time. The results of this paper have been announced, without proof, in [16].
\end{abstract}

Texte reçu le 6 juillet 2000, accepté le 25 octobre 2000

Alessandro Savo, Dipartimento di Metodi e Modelli Matematici, Università di Roma "La Sapienza", Via Antonio Scarpa 16, 00161 Roma • E-mail : savo@dmmm.uniroma1.it 2000 Mathematics Subject Classification. - 58J35, 58J50, 35P15.

Key words and phrases. - Distance function, eigenvalues of the Laplace operator, heat equation, asymptotic expansions.

I wish to thank Sylvestre Gallot, for explaining to me his ideas on symmetrization, and Gerard Besson, who made useful comments on several parts of the paper. Work partially supported by MURST and GNSAGA of Italy. 
RÉSUMÉ (Un lemme de valeur moyenne et quelques applications)

On contrôle l'écart entre la valeur moyenne d'une fonction sur une sous-variété d'une variété riemannienne, et sa valeur moyenne sur un voisinage tubulaire autour de la sous-variété (on donne, en effet, la valeur exacte de la dérivée seconde de cet écart). On applique ensuite cette formule afin d'obtenir des théorèmes de comparaison pour les valeurs propres et les fonctions propres de l'opérateur de Laplace-Beltrami, et pour calculer les trois premiers termes du développement asymptotique relatif à un problème de diffusion de la chaleur sur les polyèdres convexes dans un espace euclidien de dimension quelconque. On donne enfin des bornes explicites des restes du développement susdit, qui sont valable pour toute valeur du temps. Les résultats de cet article ont été annoncés (sans démonstrations) dans [16].

\section{Contents}

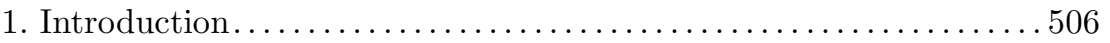

2. The mean-value lemma.......................... 511

3. Applications to eigenvalue estimates................. 517

4. Heat content asymptotics of a convex polyhedral body. . . . . . 5 523

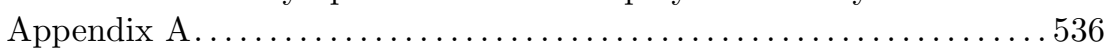

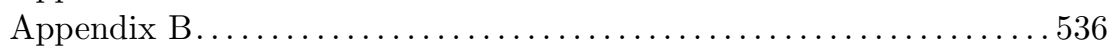

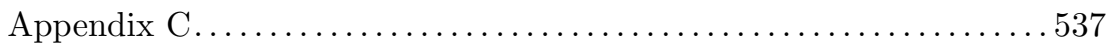

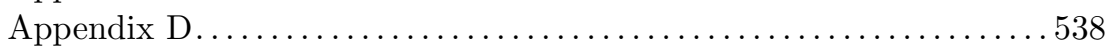

Bibliography....................................... 541

\section{Introduction}

Section 2 contains the technical background of the paper. Let $N$ be a compact, piecewise-smooth submanifold of the complete, $n$-dimensional Riemannian manifold $M$. The tube of radius $r$ around $N$ is the set

$$
M(r)=\{x \in M: \rho(x)<r\},
$$

where $\rho$ is the distance function from $N$. Given a function $u$ on $M$, our aim is to describe, in Theorem 2.5, the second derivative of the function

$$
F(r)=\int_{M(r)} u \mathrm{~d} v_{n}
$$

where $r>0$, and where $\mathrm{d} v_{n}$ is the volume form on $M$ given by the metric. This is equivalent to estimate

$$
\frac{F(r)}{\operatorname{vol}(M(r))}-\int_{N} u,
$$

TOME $129-2001-\mathrm{N}^{\mathrm{O}} 4$ 
and thus it may be seen as a generalization of the classical mean-value lemma, which says that, when $M$ is the euclidean space and $N=\left\{x_{0}\right\}$, any harmonic function satisfies $F(r) / \operatorname{vol}(M(r))=u\left(x_{0}\right)$ for all $r$.

For an arbitrary function $u$, it turns out that the second derivative of $F$ involves the Laplacian of $u$, as well as the Laplacian of the distance function $\rho$. Now, if we stay within the injectivity radius of $N$, i.e. if we stay away from the cut-locus of $N$ in $M$, both $\rho$ and $F$ will be smooth functions (of $x \in M$ and $r$ respectively); however, the nature of the problems we intend to investigate (which include the piecewise-smooth case) forced us to take into account also the points of the cut-locus, and then to consider $F(r)$ as a singular function on the whole half-line.

Due to the cut-locus, both $F$ and $\rho$ are only Lipschitz regular, and their Laplacians must therefore be taken in the sense of distributions. Hence, our first preoccupation will be to describe, in Lemma 2.1, the distributional Laplacian of the distance function, and to show that it decomposes in a regular part $\Delta_{\text {reg }} \rho$ (an $L_{\text {loc }}^{1}$-function on $M$ ), and a singular part, which is in turn the sum of a positive Radon measure $\Delta_{\text {Cut }} \rho$, supported on the cut-locus of $N$, and the Dirac measure $-2 \delta_{N}$, supported on the submanifold $N$ and vanishing when $N$ has codimension greater than 1 .

As a preparatory step, we prove a version of Green's theorem for the (generally singular) tubes $M(r)$ (Proposition 2.3); and we then proceed with the proof of the main technical lemma, called the Mean-value Lemma (see Theorem 2.5):

$$
-F^{\prime \prime}(r)=\int_{M(r)} \Delta u \mathrm{~d} v_{n}+\rho_{*}(u \Delta \rho)(r),
$$

where $\rho_{*}$ is the operator of push-forward on distributions, which is dual to the pull-back operator $\rho^{*}$. (If $r=\rho(x)$ is smaller than the injectivity radius of $N$, then $\Delta \rho$ is smooth at $x$, and gives the trace of the second fundamental form of the hypersurface $\rho^{-1}(r)$ at $x$; in that case, $\rho_{*}(u \Delta \rho)(r)=\int_{\rho^{-1}(r)} u \Delta \rho$, the integration being performed with respect to the induced measure on $\rho^{-1}(r)$ ).

Section 3 deals with the applications of Theorem 2.5 to eigenvalue estimates. Some of the results exposed here are already known, but the proofs we provide are, we believe, new, and we have chosen to include them to show the usefulness of our approach, which gives a simple unified proof of all these results. So let us select an eigenfunction $u$ of the Laplace-Beltrami operator,

$$
\Delta u=\lambda u
$$

and let

$$
F(r)=\int_{M(r)} u .
$$

Theorem 2.5 becomes the following statement:

$$
-F^{\prime \prime}=\lambda F+\rho_{*}(u \Delta \rho)
$$

BULletin DE LA SOCiÉtÉ MATHÉmATiQUe DE FRANCE 
If $u$ is harmonic, and if all the geodesic spheres of $M$ around $x_{0}$ have constant mean curvature (in particular, if $M$ is a manifold of revolution around $x_{0}$, or if $M$ is a symmetric space) then one can immediately re-derive the "classical mean-value lemma" by applying (2) in the case where $\rho$ is the distance from $x_{0}$.

The basic idea in the use of equation (2) is that it is possible to bound from below the distribution $\Delta \rho$ by an explicit radial function on $M$ (that is, a function which depends only on the distance from $N$ ), if one assumes in addition a lower bound of the Ricci curvature on $M$. Then we derive from (2) a second order differential inequality in $F$, which can be studied by standard comparison arguments. We explicitly carry out the idea in the following two cases: when $\rho$ is the distance from a point, and when $\rho$ is the distance from the boundary of a domain.

Let us apply principle (2) when $N=\left\{x_{0}\right\}$. Assume that Ricci $\geq(n-1) K$, where $K$ is any real number. Let $B\left(x_{0}, R\right)$ (resp. $\left.\bar{B}(R)\right)$ be a geodesic ball of radius $R$ in $M$ (resp. in the simply connected manifold $\bar{M}_{K}$ of constant curvature $K)$. We then obtain, in Theorem 3.1, for any positive solution of

$$
\Delta u \geq \lambda u \quad \text { on } \quad B\left(x_{0}, R\right)
$$

(resp. for any positive solution of $\Delta \bar{u}=\lambda \bar{u}$ on $\bar{B}(R)$ ), the following inequality

$$
\frac{\int_{\partial B\left(x_{0}, r\right)} u}{\int_{B\left(x_{0}, r\right)} u} \leq \frac{\int_{\partial \bar{B}(r)} \bar{u}}{\int_{\bar{B}(r)} \bar{u}}
$$

for all $0<r<R$. Theorem 3.1 reduces to the classical Bishop-Gromov inequality if $u=\bar{u}=1$. Notice that $R$ is not assumed to be smaller than the injectivity radius of $x_{0}$, so that the above inequality extends beyond the cut-locus of $x_{0}$.

We observe two consequences of Theorem 3.1: the first (Corollary 3.3), states that if $u$ is a positive superharmonic function on $B\left(x_{0}, R\right)$, then, for $0<r<R$, we have

$$
u\left(x_{0}\right) \geq \frac{1}{\operatorname{vol} \partial \bar{B}(r)} \int_{\partial B\left(x_{0}, R\right)} u,
$$

and the second (Theorem 3.4) is a well-known inequality of Cheng's regarding the first eigenvalues of the Dirichlet Laplacian on open balls in $M$ and $\bar{M}$ respectively: $\lambda_{1}(B(R)) \leq \lambda_{1}(\bar{B}(R))$ which is proved in [6], by different methods.

In the second part of Section 3, we use equation (1) in the case where $\rho$ is the distance function from the boundary of a domain $\Omega$ in $M$. We assume a lower bound $\bar{\eta}$ for the mean curvature of $\partial \Omega$, a lower bound $(n-1) K$ for the Ricci curvature of $\partial \Omega$, and we denote by $R$ the inner radius of $\Omega$ (that is, the radius of the biggest ball that fits into $\Omega$ ). We then consider the "symmetrized" domain $\bar{\Omega}$ corresponding to the data $\bar{\eta}, K, R$ : it will be the cylinder of constant curvature $K$, and width $R$, having constant mean curvature equal to $\bar{\eta}$ on

TOME $129-2001-\mathrm{N}^{\mathrm{O}} 4$ 
one, say $\Gamma$, of the two connected components of the boundary. We then show, in Theorem 3.6, that

$$
\lambda_{1}(\Omega) \geq \lambda_{1}(\bar{\Omega})
$$

where $\lambda_{1}(\Omega)$ is the first eigenvalue of the Dirichlet problem on $\Omega$, and $\lambda_{1}(\bar{\Omega})$ is the first eigenvalue of the following mixed problem on $\bar{\Omega}$ : Dirichlet condition on the component $\Gamma$, Neumann condition on the other. The result extends to any domain with piecewise-smooth boundary satisfying an additional property (see Property (P), before Lemma 3.5), and should be compared with the corresponding result obtained by Kasue [13], by different methods. In the special case $\bar{\eta}=0, K=0$, Theorem 3.6 reduces to the well-known inequality $\lambda_{1}(\Omega) \geq \pi^{2} / 4 R^{2}$, due to Li and Yau (see [15], Theorem 11).

Section 4 deals with the applications of the Mean-Value Lemma to heat diffusion. We fix a domain $\Omega$ (we assume $\partial \Omega$ piecewise-smooth), and we fix the solution $u(t, x)$ of the heat equation on $\Omega$ satisfying Dirichlet boundary conditions, and having unit initial conditions $(u(0, x)=1$ for all $x \in \Omega)$. We call $u(t, x)$ the temperature function of $\Omega$. Integrating it over $\Omega$, we obtain the heat content function $H(t)$ :

$$
H(t)=\int_{\Omega} u(t, x) \mathrm{d} x .
$$

The function $H(t)$ has been the object of investigation by a number of authors (see [1], [2], [3]); its importance lies also in the fact that, if one denotes by $k(t, x, y)$ the heat kernel of the domain $\Omega$ relative to Dirichlet boundary conditions, $H(t)$ is the integral on $\Omega \times \Omega$ of $k(t, \cdot, \cdot)$ with respect to the product measure.

Our basic idea in dealing with $H(t)$ is to introduce an auxiliary variable $r \geq 0$, and then consider the map

$$
H(t, r)=\int_{\Omega(r)} u(t, x) \mathrm{d} x,
$$

where

$$
\Omega(r)=\{x \in \Omega: d(x, \partial \Omega)>r\}
$$

are the parallel domains of $\Omega$. By the Mean-value Lemma, applied for $N=\partial \Omega$, we immediately obtain that $H(t, r)$ satisfies a heat equation on the half-line $(0, \infty)$, of the type

$$
\left(-\frac{\partial^{2}}{\partial r^{2}}+\frac{\partial}{\partial t}\right) H=-\rho_{*}(u(t, \cdot) \Delta \rho) .
$$

The main advantage of the method is that it reduces the problem to a one-dimensional one, where all computations can be performed explicitly: in fact, using Duhamel principle (Lemma 4.1), we can represent the heat content $H(t)$ in terms of the measure $\rho_{*}((1-u(t, \cdot)) \Delta \rho)$ and in terms of the Neumann 
heat kernel of the half-line, which, unlike $k(t, x, y)$, has the advantage of being explicit. One gets (see (11)):

$$
\begin{aligned}
\int_{\Omega} u(t, x) \mathrm{d} x= & \operatorname{vol}(\Omega)-\frac{2}{\sqrt{\pi}} \operatorname{vol}(\partial \Omega) \sqrt{t}+ \\
& +\int_{0}^{t} \frac{1}{\sqrt{\pi(t-\tau)}} \int_{0}^{\infty} \mathrm{e}^{-r^{2} / 4(t-\tau)} \rho_{*}((1-u(\tau, \cdot)) \Delta \rho) \mathrm{d} r \mathrm{~d} \tau .
\end{aligned}
$$

We emphasize the fact that all these computations extend beyond the cutradius and the focal radius of the normal exponential map, and therefore the estimates are valid for arbitrary values of time, and not just for small $t$ 's.

In this paper, we apply (11) to obtain the first three terms of the asymptotic expansion, for small times, of the heat content on a convex polyhedral body in the Euclidean space of arbitrary dimensions.

For a domain with smooth boundary, the third term of this expansion is

$$
\frac{1}{2}(n-1) \int_{\partial \Omega} \eta \cdot t,
$$

where $\eta$ is the mean curvature (see [1], [2], and [17] for the complete asymptotic expansion). However this term does not pass to the limit under smooth approximations of the boundary (for the unit square in the plane this term is $16 t / \pi$, while rounding off the corners a little bit one always gets $\pi t$ ). The fact is that in the polyhedral case the cut-locus hits the boundary, and then the singular part of the Laplacian of the distance function contributes with a nonneglectable term to the double integral in (11). It is exactly this contribution which we want to evaluate in this paper, leading to Theorem 4.2:

$$
\int_{\Omega} u(t, x) \mathrm{d} x=\operatorname{vol}(\Omega)-\frac{2}{\sqrt{\pi}} \operatorname{vol}(\partial \Omega) \sqrt{t}+c_{2} t+\ell(t)
$$

with

$$
c_{2}=4 \sum_{E} \operatorname{vol}_{n-2}(E) \cdot \int_{0}^{\infty}\left(1-\frac{\tanh (\gamma(E) x)}{\tanh (\pi x)}\right) \mathrm{d} x .
$$

Here $E$ runs through the set of all $(n-2)$-dimensional faces of $\partial \Omega$ (the "edges" if $\left.\Omega \subseteq \mathbb{R}^{3}\right)$, and $\gamma(E)$ is the interior angle of the two $(n-1)$-planes whose intersection is $E$. The remainder $|l(t)|$ is bounded, for all $t$, by $C t^{3 / 2}+h(t)$, for a constant $C$, and for an exponentially decreasing function $h(t)$, both explicited in $(26)$.

Theorem 4.2 generalizes to arbitrary dimension, in the convex case, the result of [3] obtained for domains in the two-dimensional plane having polygonal boundary; but in fact, if $n=2$ the constant $C$ is zero, our proof simplifies considerably, and we can extend it to cover the (not necessarily convex) polygonal case in $\mathbb{R}^{2}$ (Theorem 4.10 ).

TOME $129-2001-\mathrm{N}^{\mathrm{O}} 4$ 
We remark that formula (11) holds for both the smooth and the polyhedral case, and provides a unified approach to the problem of computing the asymptotics of the heat content in these two cases. Smooth boundaries will be in fact considered in [17], where we apply formula (11) to derive a recursive algorithm for the computation of the complete asymptotic series of the heat content, and to give sharp upper and lower bounds of the heat content in case the Ricci curvature of the domain and the mean curvature of its boundary are both non-negative (these conditions in fact guarantee that the measure $\Delta \rho$ is positive).

We sketch the main steps in the proof of Theorem 4.2.

Let then $\Omega$ be a convex polyhedral body in $\mathbb{R}^{n}$. In order to approximate the double integral in (11), one needs to accomplish two tasks:

1) Approximate the temperature function, near the boundary, by an explicit, simpler "model".

2) Control the distribution $\Delta \rho$ near the boundary.

For convex polyhedrons in a euclidean space, the regular part of the Laplacian of the distance function vanishes, i.e. $\Delta \rho$ is "purely singular", and is zero outside the cut-locus; moreover, the cut-locus is a polyhedral set itself, and we can describe $\Delta \rho$ in Proposition 4.3. The appropriate model for $u(t, \cdot)$, near an $(n-2)$-dimensional face of $\Omega$, is shown to be the temperature function on the infinite wedge in $\mathbb{R}^{n}$ bounded by the two hyperplanes which meet at the given face (this is the most delicate step in the proof). Since we only need to approximate $u$ on the cut-locus, which is contained in the bisecting plane of the wedge, we can, by a symmetry argument, reduce our calculations to the bisectrix of a wedge in the plane, and then use an explicit expression of the Laplace transform of the temperature function, due to Kontorovich and Lebedev and already employed in [3].

\section{The mean-value lemma}

Let $N$ be a smooth submanifold of the complete Riemannian manifold $M$ of dimension $n$. We assume $N$ compact. The properties of the cut-locus stated below are proved in [14] in the case $N=\left\{x_{0}\right\}$. They can be extended to arbitrary codimensions by replacing the unit sphere in the tangent space $T_{x_{0}} M$ with the unit normal bundle $U(N)$ of $N$. However, all we say in this section holds if $N$ is assumed, more generally, piecewise-smooth; we refer to the Appendix D for the extension, to the piecewise-smooth case, of all the results exposed below under the assumption of smoothness for $N$.

So let $\pi(\xi)$ be the projection of the unit vector $\xi \in U(N)$ onto its base point, and let the cut-radius $c(\xi)$ be the non-negative real number (possibly $\infty$ ), having the property that: 
the geodesic $\gamma:[0, r] \rightarrow M$ defined by $\gamma(t)=\exp _{\pi(\xi)}$ t $\xi$ minimizes the distance from $N$ if and only if $r \in[0, c(\xi)]$.

The map $c$ is then continuous from $U(N)$ to $[0, \infty]$, the 1-point compactification of $[0, \infty)$.

The cut-locus $\operatorname{Cut}(N)$ of $N$ is, by definition, the set of all points $\exp _{\pi(\xi)} c(\xi) \xi$, as $\xi$ runs through $U(N)$. Cut $(N)$ is a closed set of measure zero in M. Setting $\Phi(r, \xi)=\exp _{\pi(\xi)} r \xi$, we have a diffeomorphism from the open set

$$
U=\{(r, \xi) \in(0, \infty) \times U(N): 0<r<c(\xi)\}
$$

to $\Phi(U)=M \backslash(N \cup \operatorname{Cut}(N))$. The $(r, \xi)$ are called the normal coordinates of $M$ (based at the submanifold $N$ ). Let $\mathrm{d} v_{n}$ be the Riemannian volume form on $M$. We pull it back by the diffeomorphism $\Phi$, and we will write

$$
\Phi^{*}\left(\mathrm{~d} v_{n}\right)=\theta(r, \xi) \mathrm{d} r \mathrm{~d} \xi
$$

on $U, \theta$ being the density of the Riemannian measure in normal coordinates.

We denote by $\rho_{N}(x)$, or simply by $\rho(x)$, the distance of $x$ from $N$. The function $\rho: M \rightarrow[0, \infty)$ is Lipschitz, as it immediately follows from the triangle inequality. In normal coordinates we have simply $\rho(r, \xi)=r$, hence $\rho$, restricted to the set of its "regular points" $\Phi(U)=M \backslash(N \cup \operatorname{Cut}(N))$ is $C^{\infty}$ smooth, and, on $\Phi(U)$, we have $\|\nabla \rho\|=1$. We let

$$
\Delta_{\mathrm{reg}} \rho
$$

denote the Laplacian of $\left.\rho\right|_{\Phi(U)}$ with respect to the Riemannian metric. The following formula holds true on $U$ :

$$
\Delta_{\mathrm{reg}} \rho \circ \Phi=-\frac{1}{\theta} \frac{\partial \theta}{\partial r}
$$

For the proof, see $[10$, p. 40]. Since $\theta$ vanishes at the focal points of $N$, we see that $\Delta_{\text {reg }} \rho$ is not bounded. Nevertheless, viewed as a function on $M$ (recall that $M \backslash \Phi(U)$ has measure zero), we have:

$$
\Delta_{\mathrm{reg}} \rho \in L_{\mathrm{loc}}^{1}(M) \text {. }
$$

For the proof of this fact, see Appendix A.

The distance function $\rho$ is not, in general, $C^{1}$-smooth all over $M$, and therefore its Laplacian is not a function in the usual sense, but only a distribution; more precisely, if we define the distributional Laplacian of $\rho$ in the natural way, we have the following description of $\Delta \rho$ :

Lemma 2.1. - (i) There exists a positive distribution on $M$, denoted by $\Delta_{\mathrm{Cut}} \rho$ and supported on $\operatorname{Cut}(N)$, such that

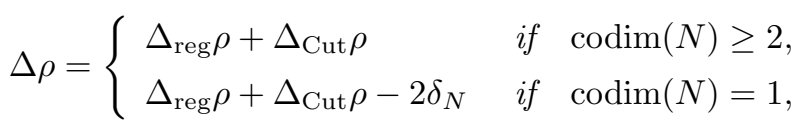

TOME $129-2001-\mathrm{N}^{\mathrm{O}} 4$ 
where $\left\langle\delta_{N}, \phi\right\rangle=\int_{N} \phi \mathrm{d} v_{n-1}$ and $\mathrm{d} v_{n-1}$ is the $(n-1)$-dimensional measure;

(ii) $\Delta \rho$ is a Radon measure, and if $\phi$ is a Lipschitz, compactly supported function on $M$ :

$$
\langle\Delta \rho, \phi\rangle=\int_{M}(\nabla \rho \cdot \nabla \phi) \mathrm{d} v_{n} .
$$

Proof. - We show that the lemma holds if we define $\Delta_{\text {Cut }} \rho$ by the formula

$$
\left\langle\Delta_{\mathrm{Cut}} \rho, \phi\right\rangle=\int_{\{\xi \in U(N): c(\xi)<\infty\}} \theta(c(\xi), \xi) \cdot \phi\left(\exp _{\pi(\xi)} c(\xi) \xi\right) \mathrm{d} v_{U(N)}(\xi),
$$

for all test-functions $\phi \in C_{c}^{\infty}(M)$, where $\theta(c(\xi), \xi)=\lim _{r \rightarrow c(\xi)-} \theta(r, \xi)$ (it is a continuous function of $\xi$ ).

Now, since $\rho$ is Lipschitz, and since $M \backslash \Phi(U)$ has measure zero, we have, by the definition of distributional Laplacian of $\rho$ :

$$
\langle\Delta \rho, \phi\rangle=\int_{M} \rho \Delta \phi=\int_{M} \nabla \rho \cdot \nabla \phi .
$$

Integrating in normal coordinates (in which $\nabla \rho=\partial / \partial r$ ):

$$
\langle\Delta \rho, \phi\rangle=\int_{U(N)} \int_{0}^{c(\xi)} \theta(r, \xi) \frac{\partial(\phi \circ \Phi)}{\partial r}(r, \xi) \mathrm{d} r \mathrm{~d} \xi .
$$

Integrating by parts in $\mathrm{d} r$, and then integrating in $\mathrm{d} \xi$, we obtain, thanks to (3) and (5):

$$
\langle\Delta \rho, \phi\rangle=\left\langle\Delta_{\text {Cut }} \rho, \phi\right\rangle+\int_{\Phi(U)}\left(\Delta_{\text {reg }} \rho\right) \phi-\int_{U(N)} \theta(0, \xi) \phi(\pi(\xi)) \mathrm{d} \xi
$$

Now the last integral is zero if $\operatorname{codim}(N) \geq 2$ (because then $\theta(0, \xi) \equiv 0$ ), and it equals $2 \int_{N} \phi \mathrm{d} v_{n-1}$ if $\operatorname{codim}(N)=1$ (because in that case $\theta(0, \xi) \equiv 1$, and $U(N)$ is locally isometric with $\left.N \times \mathbb{Z}_{2}\right)$. That $\Delta_{\text {Cut }} \rho$ is positive, and supported on $\operatorname{Cut}(N)$, is immediate from (5). Hence (i) is proved.

Proof of (ii). It follows from (i) and (5) that $\Delta \rho$ is a zero-order distribution: a classical result (see for example [19]) implies that $\Delta \rho$ is a Radon measure, as asserted. (Note in particular that $\Delta_{\text {Cut }} \rho$ is a positive Radon measure.) As regards to the last statement, first note that the formula

$$
\langle\Delta \rho, \phi\rangle=\int_{M} \nabla \rho \cdot \nabla \phi \mathrm{d} v_{n}
$$

is true if $\phi \in C_{c}^{\infty}(M)$. Since $C_{c}^{\infty}(M)$ is dense in the space of Lipschitz functions, and since both $\phi \mapsto\langle\Delta \rho, \phi\rangle$ and $\phi \mapsto \int_{M}(\nabla \rho \cdot \nabla \phi) \mathrm{d} v_{n}$ are continuous on this space, the formula extends to Lipschitz functions. The proof is complete.

The singular Laplacian of the distance function has been considered by Courtois in [8]. For the extension of Lemma 2.1 to the case where $N$ is piecewisesmooth, see Appendix D.

BULletin DE LA SOCiÉtÉ MATHÉmATIQUE DE FRANCE 
Next, we prove Green's theorem for the tubes $M(r)=\{\rho<r\}$. We use the Hausdorff measures $H_{p}$, for the definition and properties of which we refer to [5] and [9]; let us only remark here that, if $A$ is a subset of a Riemannian manifold of dimension $n$, then the $n$-dimensional Hausdorff measure of $A$ coincides with the Riemannian measure of $A$, and, in particular, if $V$ is a domain of $M$ with piecewise-smooth boundary, then $\operatorname{vol}_{n-1}(\partial V)=H_{n-1}(\partial V)$.

LEMMA 2.2. - Let $K$ be a compact subset of $M$ with $H_{n-1}(K)<\infty(n=$ $\operatorname{dim} M)$. Then there exists $\epsilon_{0}>0$ such that, for all $0<\epsilon<\epsilon_{0}$, we can find an open set $V(\epsilon)$ with piecewise smooth boundary which covers $K$, is contained in a $2 \epsilon$-neighborhood of $K$, and which satisfies:

$$
\operatorname{vol}(\partial V(\epsilon)) \leq 2^{n-1}(n-1) \cdot H_{n-1}(K)+C \epsilon,
$$

where $C$ is a positive constant which depends only on $\epsilon_{0}$ and on a lower bound of the Ricci curvature on a neighborhood of $K$.

Proof. - Appendix B.

We can now give a version of Green's theorem which will suit our needs. Given a domain $\Omega$, we will say that $\partial \Omega$ is almost regular if it is the disjoint union of two pieces $\partial_{\text {reg }} \Omega, \partial_{\text {sing }} \Omega$, where $\partial_{\text {reg }} \Omega$ is a $C^{1}$-smooth submanifold of $M$, and $\partial_{\text {sing }} \Omega$ is compact, and has zero $H_{n-1}$-measure.

Proposition 2.3. - Let $\Omega$ be a domain with almost regular boundary, and let $\nu$ denote the unit vector, normal to $\partial_{\mathrm{reg}} \Omega$ and pointing inside $\Omega$. Then if $u \in C^{2}(\Omega)$ :

$$
\int_{\Omega} \Delta u=\int_{\partial_{\mathrm{reg}} \Omega} \frac{\partial u}{\partial \nu} \mathrm{d} v_{n-1}=\int_{\partial \Omega} \frac{\partial u}{\partial \nu} \mathrm{d} H_{n-1}
$$

where $d v_{n-1}$ is the induced volume form on $\partial_{\text {reg }} \Omega$ and where $H_{n-1}$ is the Hausdorff measure.

Proof. — Fix $\epsilon>0$, and apply Lemma 2.2 to $K=\partial_{\text {sing }} \Omega$. Then

$$
\int_{\Omega} \Delta u \mathrm{~d} v_{n}=\lim _{\epsilon \rightarrow 0} \int_{\Omega \backslash(V(\epsilon) \cap \Omega)} \Delta u \mathrm{~d} v_{n} .
$$

The domain $\Omega \backslash(V(\epsilon) \cap \Omega)$ has piecewise smooth boundary given by the disjoint union of $\partial \Omega \cap V(\epsilon)^{c}$ and $\partial V(\epsilon) \cap \Omega$. Hence by the classical version of Green's theorem,

$$
\int_{\Omega \backslash(V(\epsilon) \cap \Omega)} \Delta u \mathrm{~d} v_{n}=\int_{\partial \Omega \cap V(\epsilon)^{c}} \frac{\partial u}{\partial \nu} \mathrm{d} v_{n-1}+\int_{\partial V(\epsilon) \cap \Omega} \frac{\partial u}{\partial \nu} \mathrm{d} v_{n-1} .
$$

Since $V(\epsilon)$ is contained in a $2 \epsilon$-neighborhood of $K$, we see that

$$
\int_{\partial_{\mathrm{reg}} \Omega \cap V(\epsilon)} \frac{\partial u}{\partial \nu} \mathrm{d} v_{n-1}
$$

TOME $129-2001-\mathrm{N}^{\mathrm{O}} 4$ 
tends to zero with $\epsilon$, by Lebesgue bounded convergence theorem. Therefore $\int_{\partial \Omega \cap V(\epsilon)^{c}} \partial u / \partial \nu \mathrm{d} v_{n-1}$ converges to $\int_{\partial_{\mathrm{reg}} \Omega} \partial u / \partial \nu \mathrm{d} v_{n-1}$. On the other hand, by Lemma 2.2, $\operatorname{vol}(\partial V(\epsilon)) \rightarrow 0$, and therefore $\int_{\partial V(\epsilon) \cap \Omega} \partial u / \partial \nu \mathrm{d} v_{n-1}$ converges to 0 as $\epsilon \rightarrow 0$, since $|\partial u / \partial \nu| \leq\|\nabla u\|$ is bounded.

Now fix $r>0$. We say that $r$ is a regular value of $\rho$ if

$$
H_{n-1}\left(\rho^{-1}(r) \cap \operatorname{Cut}(N)\right)=0 .
$$

We see immediately that, if $r$ is a regular value of $\rho$, then $\partial M(r)=\rho^{-1}(r)$ is almost regular, with $\partial_{\text {reg }} \Omega=\rho^{-1}(r) \cap \Phi(U)$, and $\partial_{\text {sing }} \Omega=\rho^{-1}(r) \cap \operatorname{Cut}(N)$. Since $\operatorname{Cut}(N)$ has zero measure, we have, as a consequence of Eilenberg's inequality (see [5, Thm 13.3.1]), that the complement of the set of regular values of $\rho$ has zero Lebesgue measure. Therefore, for almost all $r \in(0, \infty), \partial M(r)$ is almost regular; and since $\nabla \rho$ coincides with the unit normal to $\partial_{\text {reg }} M(r)$, pointing outside $M(r)$, we have, for all $u \in C^{2}(M)$ and for almost all $r$ :

$$
\int_{M(r)} \Delta u \mathrm{~d} v_{n}=-\int_{\rho^{-1}(r)}(\nabla u \cdot \nabla \rho) \mathrm{d} H_{n-1} .
$$

Now fix $u \in C^{2}(M)$, and consider the function $F:(0, \infty) \rightarrow \mathbb{R}$ defined by

$$
F(r)=\int_{M(r)} u \mathrm{~d} v_{n}
$$

where $M(r)$ is, as usual, the tube of radius $r$ around $N$. If $N$ is smooth, then $F$ is smooth on $\left(0, R_{\mathrm{inj}}\right)$, where $R_{\mathrm{inj}}$ is the injectivity radius of the normal exponential map of $N$. In the general case, we have:

Lemma 2.4. - F is Lipschitz on each compact interval and, for almost all $r \in(0, \infty)$ :

$$
F^{\prime}(r)=\int_{\rho^{-1}(r) \cap \Phi(U)} u \mathrm{~d} v_{n-1} .
$$

Moreover, the map $r \mapsto \int_{\rho^{-1}(r) \cap \Phi(U)} u \mathrm{~d} v_{n-1}$ is continuous from the right on $(0, \infty)$.

Proof. - From the formula of co-area (see [5, Cor. 13.4.6]):

$$
F(r)=\int_{0}^{r} \int_{\rho^{-1}(s)} u \mathrm{~d} H_{n-1} \mathrm{~d} s .
$$

Hence

$$
F^{\prime}(r)=\int_{\rho^{-1}(r)} u \mathrm{~d} H_{n-1}
$$

a.e. on $(0, \infty)$, and in turn $F^{\prime}(r)=\int_{\rho^{-1}(r) \cap \Phi(U)} u \mathrm{~d} v_{n-1}$ a.e. on $(0, \infty)$ (precisely, on the set of regular values of $\rho$ ). 
Now observe that

$$
\int_{\rho^{-1}(r) \cap \Phi(U)} u \mathrm{~d} v_{n-1}=\int_{U(N)} f_{r}(\xi) \mathrm{d} \xi
$$

where $f_{r}(\xi)=u(\Phi(r, \xi)) \theta(r, \xi)$ if $c(\xi)>r$ and $f_{r}(\xi)=0$ if $c(\xi) \leq r$. Since $\theta(r, \xi)$ is uniformly bounded in $r \in[a, b]$ (and $\xi \in U(N))$ by Rauch's comparison theorem, so is $\int_{\rho^{-1}(r) \cap \Phi(U)} u \mathrm{~d} v_{n-1}$, hence $F$ is Lipschitz on $[a, b]$. Finally, if $r_{n} \downarrow r$, then, for $\xi$ fixed, $f_{r_{n}}(\xi) \rightarrow f_{r}(\xi)$ and the last assertion follows by Lebesgue's bounded convergence theorem.

We now come to the computation of $F^{\prime \prime}$. Let $\psi \in C_{c}^{0}(0, \infty)$. Since $\rho$ is a proper map, the pull back $\psi \circ \rho$ is a continuous, compactly supported function on $M$. Hence if $T$ is a Radon measure on $M$, its push-forward $\rho_{*}(T)$ exists and is the measure on $(0, \infty)$ defined by the relation

$$
\left\langle\rho_{*}(T), \psi\right\rangle=\langle T, \psi \circ \rho\rangle .
$$

Note that, if $u$ is a function on $M$, then, by the co-area formula, $\rho_{*}(u)$ is the regular distribution defined a.e. by $\rho_{*}(u)(r)=\int_{\rho^{-1}(r)} u \mathrm{~d} H_{n-1}=F^{\prime}(r)$ (see Lemma 2.4).

We can now state our main technical lemma.

TheOREm 2.5 (Mean-value Lemma). - Let $\rho: M \rightarrow[0, \infty)$ be the function: distance from $N$, where $N$ is a compact, piecewise-smooth submanifold of $M$; let $u \in C^{2}(M)$, and $M(r)=\{x \in M: \rho(x)<r\}$. If $F(r)=\int_{M(r)} u \mathrm{~d} v_{n}$, then we have, as Radon measures on $(0, \infty)$ :

$$
-F^{\prime \prime}(r)=\int_{M(r)} \Delta u \mathrm{~d} v_{n}+\rho_{*}(u \Delta \rho)(r) .
$$

Proof. - It is enough to verify the equality when both sides are tested on a smooth, compactly supported function $\psi$ on $(0, \infty)$. Then, by Lemma 2.4:

$$
-\left\langle F^{\prime \prime}, \psi\right\rangle=\int_{0}^{\infty} F^{\prime} \psi^{\prime}=\int_{0}^{\infty} \int_{\rho^{-1}(r)} u\left(\psi^{\prime} \circ \rho\right) \mathrm{d} H_{n-1} \mathrm{~d} r=\int_{M} u\left(\psi^{\prime} \circ \rho\right) .
$$

The last equality uses co-area formula. Now, on the set of regular points $\Phi(U)$, (hence a.e. on M) the map $\psi \circ \rho$ is $C^{\infty}$ and we have $\nabla(\psi \circ \rho)=\left(\psi^{\prime} \circ \rho\right) \nabla \rho$. Hence:

$-\left\langle F^{\prime \prime}, \psi\right\rangle=\int_{M} u(\nabla(\psi \circ \rho) \cdot \nabla \rho)=\int_{M} \nabla(u(\psi \circ \rho)) \cdot \nabla \rho-\int_{M}(\psi \circ \rho)(\nabla u \cdot \nabla \rho)$.

Since $\psi \circ \rho$ is Lipschitz, we have, by Lemma 2.1 (ii), that the first term is equal to $\langle\Delta \rho, u(\psi \circ \rho)\rangle$, and then, by the definition of push-forward, also equal to $\left\langle\rho_{*}(u \Delta \rho), \psi\right\rangle$. The second term is equal to

$$
-\int_{0}^{\infty} \psi \int_{\rho^{-1}(r)}(\nabla u \cdot \nabla \rho) \mathrm{d} H_{n-1} \mathrm{~d} r
$$

TOME $129-2001-\mathrm{N}^{\mathrm{O}} 4$ 
by the co-area formula, and then, thanks to formula 6 , also equal to $\int_{0}^{\infty} \psi\left(\int_{M(r)} \Delta u\right) \mathrm{d} r$. The proof is complete.

An important particular case is when $N$ is the boundary of a domain $\Omega$ in $M$; for our convenience, we then restrict $\rho$ to $\Omega$, and consider $\Delta \rho$ as a distribution on $\Omega$. Changing $M(r)$ in $\Omega(r)=\{x \in \Omega: \rho(x)>r\}$, and the previous definition of $F(r)$ in $F(r)=\int_{\Omega(r)} u \mathrm{~d} v_{n}$, we obtain the following version of the Mean-value Lemma:

$$
-F^{\prime \prime}(r)=\int_{\Omega(r)} \Delta u \mathrm{~d} v_{n}-\rho_{*}(u \Delta \rho),
$$

as Radon measures on $(0, \infty)$. For the proof of $(7)$, just repeat the proof of Theorem 2.5, with the indicated changes; and observe that now $F^{\prime}(r)=$ $-\int_{\rho^{-1}(r)} u \mathrm{~d} H_{n-1}($ a.e. on $(0, \infty))$.

REMARK 2.6. - We can replace $u$ in Theorem 2.5 (or in its special case 7) by any continuous, compactly supported function on $M$ having the property that $\Delta u$ is a Radon measure.

\section{Applications to eigenvalue estimates}

3.1. Applications when $\rho$ is the distance from a point. - Let $M$ be a manifold on which we make the following curvature assumptions

$$
\text { Ricci } \geq(n-1) K,
$$

where $K$ can assume all real values. Let $\theta=\theta(r, \xi)$ denote, as before, the density of the Riemannian measure in normal (polar) coordinates centered at a given point $x_{0} \in M$, and let $\bar{\theta}$ be the corresponding density, relative to a given point $\bar{x}_{0}$, on the simply connected manifold $\bar{M}_{K}$ of constant curvature $K$. By Bishop comparison theorem (see [4]), we have $\theta^{\prime} / \theta(r, \xi) \leq \bar{\theta}^{\prime} / \bar{\theta}(r)$ for all $(r, \xi) \in U$ and therefore

$$
\Delta_{\text {reg }} \rho \geq-\frac{\bar{\theta}^{\prime}}{\bar{\theta}} \circ \rho
$$

at all regular points of $\rho$ (see (3)).

We will be dealing with the integral of a function on geodesic spheres centered at $x_{0}$ in $M$. We point out the fact that, when $r>R_{\text {inj }}\left(x_{0}\right), \partial B\left(x_{0}, r\right)$ is no longer a regular submanifold of $M$; however, we can integrate a function on the "regular part" of it: $\partial_{\text {reg }} B\left(x_{0}, r\right) \equiv \partial B\left(x_{0}, r\right) \cap \Phi(U)$.

Hence, in this section, we agree to set, for all $r$

$$
\int_{\partial B\left(x_{0}, r\right)} u \equiv \int_{\partial_{r e g} B\left(x_{0}, r\right)} u \mathrm{~d} v_{n-1} .
$$

BULletin DE LA SOCiÉtÉ MATHÉmATiQUe DE FRANCE 
Recall that $\int_{\partial B_{\text {reg }}\left(x_{0}, r\right)} u \mathrm{~d} v_{n-1}$ does coincide with $\int_{\partial B\left(x_{0}, r\right)} u \mathrm{~d} H_{n-1}$ on the set of regular values $r$ of $\rho$, hence a.e. on $(0, \infty)$ (see $\S 2)$.

We can now state the main result of this subsection, which is a comparison theorem between solutions of the equation $\Delta u=\lambda u$ on $M$ and $\bar{M}_{K}$.

THEOREM 3.1. - Let $M$ be a manifold satisfying Ricci $\geq(n-1) K$, and let $\lambda \in \mathbb{R}$, and $R \leq \operatorname{diam}(M)$. Assume that $u$ is positive on the open ball $B\left(x_{0}, R\right)$ in $M$ and satisfies $\Delta u \geq \lambda u$, and let $\bar{u}$ be a solution of $\Delta \bar{u}=\lambda \bar{u}$ on the ball $B\left(\bar{x}_{0}, R\right) \equiv \bar{B}(R)$ in $\bar{M}_{K}$ such that $\bar{u}\left(\bar{x}_{0}\right) \neq 0$. Then, for all $r \leq R$ :

$$
\frac{\int_{\partial B\left(x_{0}, r\right)} u}{\int_{B\left(x_{0}, r\right)} u} \leq \frac{\int_{\partial \bar{B}(r)^{\bar{u}}} \overline{\int_{\bar{B}(r)}}}{\int} \text { and } \frac{1}{u\left(x_{0}\right)} \int_{B\left(x_{0}, r\right)} u \leq \frac{1}{\bar{u}\left(\bar{x}_{0}\right)} \int_{\bar{B}(r)} \bar{u} .
$$

Let us notice that in Theorem 3.1 we don't assume any boundary conditions for the functions $u$ and $\bar{u}$. About the existence of solutions of $\Delta \bar{u}=\lambda \bar{u}$ on the space form $\bar{M}_{K}$, we have the following:

Lemma 3.2. - Let $\lambda \in \mathbb{R}, \bar{x}_{0} \in \bar{M}_{K}, R \leq \operatorname{diam}\left(\bar{M}_{K}\right)$. Then there exists a unique radial solution of $\Delta \bar{u}=\lambda \bar{u}$ on the open ball $B\left(\bar{x}_{0}, R\right)$, having a preassigned value at $\bar{x}_{0}$. Here "radial" means that there exists a function $f:[0, R) \rightarrow \mathbb{R}$ such that $\bar{u}=f \circ \rho$, where $\rho=d\left(\bar{x}_{0}, \cdot\right)$ in $\bar{M}_{K}$.

Proof. - Since $\Delta(f \circ \rho)=-\left(f^{\prime \prime}+\bar{\theta}^{\prime} / \bar{\theta} f^{\prime}\right) \circ \rho$, solving the equation $\Delta \bar{u}=\lambda \bar{u}$ on $B\left(\bar{x}_{0}, R\right)$ amounts to solve the equation: $\bar{\theta} f^{\prime \prime}+\bar{\theta}^{\prime} f^{\prime}+\lambda \bar{\theta} f=0$ on the interval $(0, R)$. The assertion now follows from the theory of second order differential equations (see for example [7]), and the regularity of $\bar{u}$ which implies $f^{\prime}(0)=0$.

Proof of Theorem 3.1. - We can assume that $\bar{u}\left(\bar{x}_{0}\right)>0$. Let $F(r)=$ $\int_{B\left(x_{0}, r\right)} u$, and fix a small $\epsilon>0$. By Theorem 2.5 and by Lemma 2.1 (i), we have, as measures on $(\epsilon, R)$ :

$$
-F^{\prime \prime} \geq \lambda F+\int_{\partial B\left(x_{0}, r\right)} u \Delta_{\mathrm{reg}} \rho+\rho_{*}\left(u \Delta_{\mathrm{Cut}} \rho\right)
$$

and then, from (8), the positivity of $\Delta_{\mathrm{Cut}} \rho$, and Lemma2.4:

$$
F^{\prime \prime}-\frac{\bar{\theta}^{\prime}}{\bar{\theta}} F^{\prime}+\lambda F \leq 0 .
$$

On the other hand, the corresponding map $\bar{F}(r)=\int_{\bar{B}(r)} \bar{u}$ satisfies, on $(\epsilon, R)$, the equation:

$$
\bar{F}^{\prime \prime}-\frac{\bar{\theta}^{\prime}}{\bar{\theta}} \bar{F}^{\prime}+\lambda \bar{F}=0 .
$$

In fact, on $\bar{M}_{K}$ the cut-locus of any point reduces to a single point or is empty, so that $\Delta_{\text {Cut }} \rho=0$; and as $\Delta_{\text {reg }} \rho=-\bar{\theta}^{\prime} / \bar{\theta} \circ \rho$, we have (10) by the

TOME $129-2001-\mathrm{N}^{\mathrm{O}} 4$ 
Mean-value Lemma. Now let $R_{0}$ be the first zero of $\bar{F}$, so that $\bar{F} \geq 0$ on $\left(\epsilon, R_{0}\right)$, and let $R_{1}=\min \left\{R_{0}, R\right\}$. We multiply $(9)$ by $\bar{F},(10)$ by $F$ and add. Then, on $\left(\epsilon, R_{1}\right)$, we have the inequality $\left(F^{\prime} \bar{F}-\bar{F}^{\prime} F\right)^{\prime}-\bar{\theta}^{\prime} / \bar{\theta}\left(F^{\prime} \bar{F}-\bar{F}^{\prime} F\right) \leq 0$ so that, if

$$
W(r)=\frac{F^{\prime}(r) \bar{F}(r)-\bar{F}^{\prime}(r) F(r)}{\bar{\theta}(r)},
$$

then $W^{\prime}$ is a negative measure on $\left(\epsilon, R_{1}\right)$. It is a well-known fact that then $W^{\prime}=d g$ for a monotone decreasing function $g$; since $W=g$ on a dense subset of $\left(\epsilon, R_{1}\right)$, and since $W$ is continuous from the right (by Lemma 2.4), $W$ is itself decreasing on $\left(0, R_{1}\right)$. Now $W(\epsilon)$ tends to zero with $\epsilon$, and we obtain the first inequality of the theorem with $R_{1}$ replacing $R$. Next, we integrate both sides of $F^{\prime} / F \leq \bar{F}^{\prime} / \bar{F}$ from $\epsilon$ to $r$, and get

$$
\frac{F(r)}{\bar{F}(r)} \leq \frac{F(\epsilon)}{\bar{F}(\epsilon)}
$$

but since $\operatorname{vol}\left(B\left(x_{0}, \epsilon\right)\right) / \operatorname{vol}(\bar{B}(\epsilon)) \rightarrow 1$ when $\epsilon \rightarrow 0$, we see that the limit inequality is $F(r) / \bar{F}(r) \leq u\left(x_{0}\right) / \bar{u}\left(\bar{x}_{0}\right)$, which is precisely the second inequality with $R_{1}$ replacing $R$. It then remains to show that $R_{1}=R$, or that $R_{0} \geq R$. Assume not. Then we would have $0<F\left(R_{0}\right) \leq u\left(x_{0}\right) / \bar{u}\left(\bar{x}_{0}\right) \bar{F}\left(R_{0}\right)=0$. The proof is complete.

We observe that, for $u=1$, the theorem reduces to the well-known BishopGromov inequality.

Corollary 3.3. - Assume Ricci $\geq(n-1) K$. If $u$ is a positive superharmonic function on $B\left(x_{0}, R\right)$ (i.e. $\Delta u \geq 0$ ) then, for all $r \leq R$, we have:

$$
u\left(x_{0}\right) \geq \frac{1}{\operatorname{vol}(\partial \bar{B}(r))} \int_{\partial B\left(x_{0}, r\right)} u .
$$

Another application of Theorem 3.1 is a new proof of the following result of Cheng (see [6]) on the first eigenvalue of the Laplace-Beltrami operator on geodesic balls. Let us denote by $\lambda_{1}(\Omega)$ the first non-zero eigenvalue of the Dirichlet problem on $\Omega$.

Theorem 3.4 (Cheng). - If Ricci $\geq(n-1) K$, then, for all $R$ :

$$
\lambda_{1}\left(B\left(x_{0}, R\right)\right) \leq \lambda_{1}(\bar{B}(R))
$$

where $\bar{B}(R)$ is the ball of radius $R$ in the simply connected manifold of constant sectional curvature $K$.

Proof. - Let us assume that $\lambda_{1}\left(B\left(x_{0}, R\right)\right)>\lambda_{1}(\bar{B}(R))$. Then there exists $R^{\prime}<R$ such that $\lambda_{1}\left(B\left(x_{0}, R\right)\right)=\lambda_{1}\left(\bar{B}\left(R^{\prime}\right)\right)$. Choose corresponding positive eigenfunctions $u$ (resp. $\bar{u}$ ) on $B\left(x_{0}, R\right)$ (resp. $\left.\bar{B}\left(R^{\prime}\right)\right)$. The positivity of $u$ in 
the interior of $B\left(x_{0}, R\right)$ implies that $\int_{\partial B\left(x_{0}, R^{\prime}\right)} u>0$; as $\bar{u} \equiv 0$ on $\partial \bar{B}\left(R^{\prime}\right)$, this is a contradiction with Theorem 3.1.

3.2. Applications when $\rho$ is the distance from the boundary of a domain. - In this subsection we give a lower bound for the first eigenvalue of the Dirichlet Laplacian of a relatively compact domain $\Omega$ having smooth boundary, or piecewise-smooth boundary satisfying an additional condition (see Property (P) below). Denote by

$$
\rho: \Omega \longrightarrow \mathbb{R}
$$

the distance function from the boundary of $\Omega$. Then we have, as distributions on $\Omega$ (i.e. as continuous linear maps on $C_{c}^{\infty}(\Omega)$ )

$$
\Delta \rho=\Delta_{\mathrm{reg}} \rho+\Delta_{\mathrm{Cut}} \rho,
$$

where $\Delta_{\text {Cut }} \rho$ is positive, and supported on the cut-locus of $\partial \Omega$. Let us write

$$
\partial \Omega=\partial_{\text {reg }} \Omega \cup \partial_{\text {sing }} \Omega
$$

where $\partial_{\text {reg }} \Omega$ is a smooth submanifold of codimension 1 and $\partial_{\text {sing }} \Omega$ is the singular part of $\partial \Omega$.

We will say that $\Omega$ satisfies property $(\mathrm{P})$ if

For each $x \in \Omega \backslash \operatorname{Cut}(\partial \Omega)$ the foot of the geodesic segment which minimizes the distance from $x$ to $\partial \Omega$ is a regular point of $\partial \Omega$.

For example, a two-dimensional domain satisfies $(\mathrm{P})$ if and only if the interior angle at any of the singular points of the boundary is convex. In fact, the condition $(\mathrm{P})$ is imposed to insure that the mean curvature of the level sets does not become too negative near the singularities of the boundary.

Under the assumption $(\mathrm{P})$, we then have

$$
\Delta_{\mathrm{reg}} \rho=-\frac{\theta^{\prime}}{\theta} \circ \rho
$$

where $\theta$ is the Jacobian of the diffeomorphism (normal chart)

$$
\Phi: U \longrightarrow \Omega \backslash \operatorname{Cut}(\partial \Omega)
$$

which sends $(r, \xi)$ to $\exp _{\pi(\xi)} r \xi$. Here

$$
U=\left\{(r, \xi) \in(0, \infty) \times U\left(\partial_{\mathrm{reg}} \Omega\right): 0<r<c(\xi), \Phi(r, \xi) \in \Omega\right\} .
$$

If $\rho$ is smooth at $x$, and if $\rho(x)=r$, then $\Delta_{\text {reg }} \rho$ gives the trace of the second fundamental form of the level submanifold $\rho^{-1}(r)$ at $x$; the mean curvature is then defined as $1 /(n-1) \Delta_{\text {reg }} \rho(x)$ (our sign convention is that the mean curvature of the unit sphere in euclidean space is positive).

TOME $129-2001-\mathrm{N}^{\mathrm{O}} 4$ 
LEMMA 3.5. - Let $\Omega$ be a relatively compact, open set of $M$, with piecewise smooth boundary satisfying property $(\mathrm{P})$. Assume that the mean curvature of $\partial_{\text {reg }} \Omega$ is bounded below by $\bar{\eta}$, and that Ricci $\geq(n-1) K$ on $\Omega$. Then, as distributions on $\Omega$ :

$$
\Delta \rho \geq-\frac{\bar{\theta}^{\prime}}{\bar{\theta}} \circ \rho
$$

where

and where

$$
\bar{\theta}(r)=\left(s_{K}^{\prime}(r)-\bar{\eta} s_{K}(r)\right)^{n-1}
$$

$$
s_{K}(r)= \begin{cases}\frac{1}{\sqrt{K}} \sin (r \sqrt{K}) & \text { if } \quad K>0, \\ r & \text { if } \quad K=0, \\ \frac{1}{\sqrt{|K|}} \sinh (r \sqrt{|K|}) & \text { if } \quad K<0 .\end{cases}
$$

In particular, if $\Omega$ satisfies property $(\mathrm{P})$, and if the mean curvature of the regular part of the boundary and the Ricci curvature of $\Omega$ are both non-negative, then $\Delta \rho \geq 0$.

Proof. - Since $\Delta_{\text {Cut }} \rho \geq 0$, it is enough to show that $\Delta_{\text {reg }} \rho \geq-\bar{\theta}^{\prime} / \bar{\theta} \circ \rho$. This fact is a consequence of (3) and Heintze-Karcher's estimates in [12], or else it can be re-derived by the same procedure followed in $[10, \mathrm{p} .41]$. In the last statement, we suppose that $K=0$ and $\bar{\eta}=0$, so the fact that $-\bar{\theta}^{\prime} / \bar{\theta}=$ $(n-1) \bar{\eta} /(1-r \bar{\eta}) \geq 0$ implies that $\Delta \rho \geq 0$.

To state our comparison theorem, we need to define the model domains to which we will compare our domain $\Omega$. Then let

$$
\bar{\Omega} \equiv \bar{\Omega}(K, \bar{\eta}, R)
$$

be the cylinder with constant curvature $K$, and width $R$, such that the mean curvature is constant, equal to $\bar{\eta}$, on one of the two connected components of the boundary. Depending on $K$ and $\bar{\eta}, \Omega$ will be an annulus in either the space form $\bar{M}_{K}$, or the hyperbolic cylinder of constant curvature $K$. We postpone the explicit realization of $\bar{\Omega}$ after we have proved the following comparison theorem.

THEOREM 3.6 (compare with [13]). - Let $\Omega$ be a domain with piecewise smooth boundary satisfying property $(\mathrm{P})$. Assume that the Ricci curvature of $\Omega$ is bounded below by $(n-1) K$, that the mean curvature of $\partial_{\text {reg }} \Omega$ is bounded below by $\bar{\eta}$, and let $R$ be the inner radius of $\Omega$. Then:

$$
\lambda_{1}(\Omega) \geq \bar{\lambda}_{1}(\bar{\Omega}),
$$

where $\lambda_{1}$ is the first non-zero eigenvalue of the Dirichlet problem on $\Omega$, and where $\bar{\lambda}_{1}(\bar{\Omega})$ denotes the first non-zero eigenvalue of the following mixed problem on $\bar{\Omega}(K, \bar{\eta}, R)$ : Dirichlet condition on the component having mean curvature $\bar{\eta}$, Neumann condition on the other. 
Proof. - Let $\bar{\rho}: \bar{\Omega} \rightarrow(0, \infty)$ denote the distance function from $\Gamma$, the component of $\partial \bar{\Omega}$ having constant mean curvature $\bar{\eta}$. From the explicit expression of $\bar{\Omega}$, it will be clear that the cut-locus of $\Gamma$ is either empty, or reduces to a point: hence $\Delta_{\text {Cut }} \bar{\rho}=0$; moreover $\Delta \bar{\rho}=\Delta_{\text {reg }} \bar{\rho}=-\bar{\theta}^{\prime} / \bar{\theta} \circ \bar{\rho}$ where $\bar{\theta}$ is as in Lemma 3.5. Let $u$ be a positive eigenfunction corresponding to $\lambda=\lambda_{1}(\Omega)$, let $\bar{u}$ be the eigenfunction associated to $\bar{\lambda}=\bar{\lambda}_{1}(\bar{\Omega})$ which is positive on $\bar{\Omega}$ and is normalized so that $\int_{\bar{\Omega}} \bar{u}=\int_{\Omega} u$, and let

$$
F(r)=\int_{\Omega(r)} u \text { and } \bar{F}(r)=\int_{\bar{\Omega}(r)} \bar{u} .
$$

By the version (7) of the Mean-value Lemma, by Lemma 3.5, and the fact that $\rho_{*}(u)(r)=-F^{\prime}(r)$, and $\bar{\rho}_{*}(\bar{u})(r)=-\bar{F}^{\prime}(r)$ (see Lemma 2.4), we see that, in the sense of distributions:

$$
F^{\prime \prime}-\frac{\bar{\theta}^{\prime}}{\bar{\theta}} F^{\prime}+\lambda F \geq 0, \quad \bar{F}^{\prime \prime}-\frac{\bar{\theta}^{\prime}}{\bar{\theta}} \bar{F}^{\prime}+\bar{\lambda} \bar{F}=0 .
$$

Assume $\lambda<\bar{\lambda}$. We multiply the first inequality by $\bar{F}$, the second equation by $F$, and subtract. We get

$$
\left(\frac{F^{\prime} \bar{F}-F \bar{F}^{\prime}}{\bar{\theta}}\right)^{\prime} \geq \frac{F \bar{F}(\bar{\lambda}-\lambda)}{\bar{\theta}}>0 .
$$

Hence $\left(F^{\prime} \bar{F}-F \bar{F}^{\prime}\right) / \bar{\theta}>\left(F^{\prime}(0) \bar{F}(0)-F(0) \bar{F}^{\prime}(0)\right) / \bar{\theta}(0)=0$, which implies: $F^{\prime}(r) / F(r)>\bar{F}^{\prime}(r) / \bar{F}(r)$ on $(0, R)$. By our normalization $(F(0)=\bar{F}(0))$ we obtain $F(r)>\bar{F}(r)$, and, in turn $F^{\prime}(r)>\bar{F}^{\prime}(r)$ on $(0, R)$. Ultimately we would have

$$
F(0)=-\int_{0}^{R} F^{\prime}(r) \mathrm{d} r<-\int_{0}^{R} \bar{F}^{\prime}(r) \mathrm{d} r=\bar{F}(0)
$$

which is a contradiction. Hence $\lambda \geq \bar{\lambda}$.

We now proceed to the explicit construction of the model cylinder

$$
\bar{\Omega}=\bar{\Omega}(K, \bar{\eta}, R) .
$$

CASE 1. $-K>0, \bar{\eta} \in \mathbb{R}$, or $K<0,|\bar{\eta}|>\sqrt{|K|}$, or $K=0, \bar{\eta} \in \mathbb{R} \backslash\{0\}$.

Let us denote by $B_{K}(r)$ the geodesic ball of radius $r$ (centered at some fixed origin point $x_{0}$ ) in the simply connected space of constant curvature $K$. In normal coordinates around $x_{0}$, the metric is written

$$
\bar{g}=d r^{2}+s_{K}^{2}(r) \cdot g_{S^{n-1}},
$$

where $s_{K}(r)$ is defined in Lemma 3.5. Let us set

$$
\cot _{K}(r)=\frac{s_{K}^{\prime}(r)}{s_{K}(r)} \quad \text { and } \quad \bar{R}=\cot _{K}^{-1}(|\bar{\eta}|),
$$

TOME $129-2001-\mathrm{N}^{\mathrm{O}} 4$ 
which is defined for any value of $\bar{\eta}$ when $K>0$, for $\bar{\eta} \neq 0$ when $K=0$, and for $|\bar{\eta}|>\sqrt{|K|}$ when $K<0$. As we already remarked, the inner radius $R$ of $\Omega$ is bounded above by $\bar{R}$, and we may take:

$$
\bar{\Omega}=\left\{\begin{array}{lll}
B_{K}(\bar{R}) \backslash B_{K}(\bar{R}-R) & \text { if } \quad \bar{\eta} \geq 0, \\
B_{K}(\bar{R}+R) \backslash B_{K}(\bar{R}) & \text { if } \quad \bar{\eta}<0 .
\end{array}\right.
$$

In both cases, $\Gamma=\partial B_{K}(\bar{R})$, viewed as the boundary of $\bar{\Omega}$, has constant mean curvature $\bar{\eta}$.

CASE 2. $-K=0, \bar{\eta}=0$, or $K<0, \bar{\eta} \in(-\sqrt{|K|}, \sqrt{|K|})$.

Set $t_{K}(r)=s_{K}^{\prime \prime}(r) / s_{K}^{\prime}(r)$, and let $\tilde{R}=0$ in the first case, $\tilde{R}=t_{K}^{-1}(\bar{\eta})$ in the second case. We take $\bar{\Omega}=(\tilde{R}-R, \tilde{R}) \times S^{n-1}$ and we endow it with the metric $\bar{g}=d r^{2}+s_{K}^{\prime}(r)^{2} \cdot g_{S^{n-1}}$. In both cases the component $\Gamma=\{\tilde{R}\} \times S^{n-1}$ of $\partial \bar{\Omega}$ has mean curvature $t_{K}(\tilde{R})=\bar{\eta}$.

CASE 3. $-K<0, \bar{\eta}= \pm \sqrt{|K|}$.

These are the limit cases of Case 1 as $\bar{R} \rightarrow \infty$.

REMARK 3.7. - We observe that when both the Ricci curvature of $\Omega$ and the mean curvature of $\partial \Omega$ are non-negative, then $\bar{\Omega}=(0, R) \times S^{n-1}$ and Theorem 3.6 reduces the the inequality $\lambda_{1}(\Omega) \geq \pi^{2} /\left(4 R^{2}\right)$ due to Li and Yau (Theorem 11 in [15]).

\section{Heat content asymptotics of a convex polyhedral body}

Let $\Omega$ be an open set with compact closure and piecewise-smooth boundary in a complete Riemannian manifold $M$, and let $w(t, x)$ be any solution of the heat equation on $\Omega$. Consider the function $f:(0, \infty) \times[0, \infty) \rightarrow \mathbb{R}$ defined by

$$
f(t, r)=\int_{\Omega(r)} w(t, x) \mathrm{d} x,
$$

where $\Omega(r)=\{x \in \Omega: \rho(x)>r\}$, and where $\rho$ is the distance function from the boundary.

It is an immediate consequence of the mean-value lemma (formula (7)) that $f(t, r)$ satisfies the following heat equation on the half-line

$$
\left(-\frac{\partial^{2}}{\partial r^{2}}+\frac{\partial}{\partial t}\right) f=-\rho_{*}(w(t, \cdot) \Delta \rho) .
$$


LEMMA 4.1 (Duhamel principle). - Let $f(t, r)$ be as above. Then, for all $t>0$ and $r \geq 0$, we have:

$$
\begin{aligned}
f(t, r)=\int_{0}^{\infty} & e(t, r, s) f(0, s) d s-\int_{0}^{t} \int_{0}^{\infty} e(t-\tau, r, s) \rho_{*}((w(\tau, \cdot) \Delta \rho)(s) \mathrm{d} s \mathrm{~d} \tau \\
& -\int_{0}^{t} \frac{\partial f}{\partial r}(\tau, 0) e(t-\tau, r, 0) \mathrm{d} \tau
\end{aligned}
$$

where $e(t, r, s)$ denotes the heat kernel of the half-line relative to Neumann boundary conditions at 0 , which is given by the explicit formula

$$
e(t, r, s)=\frac{1}{\sqrt{4 \pi t}}\left(\mathrm{e}^{(r-s)^{2} / 4 t}+\mathrm{e}^{(r+s)^{2} / 4 t}\right)
$$

for all $t>0, r, s>0$.

Proof. - $\partial^{2} f / \partial r^{2}(\tau, \cdot)$ is a measure on $(0, \infty)$, and we can integrate by parts twice in $\int_{0}^{\infty} e(t-\tau, r, s) \partial^{2} f / \partial r^{2}(\tau, s) \mathrm{d} s$. Therefore the classical proof applies without change.

We will use Duhamel principle to study the heat content function

$$
H(t)=\int_{\Omega} u(t, x) \mathrm{d} x,
$$

where $u(t, x)$ is the solution of the heat equation on $\Omega$, with Dirichlet boundary conditions, and with unit initial conditions: $u(0, x)=1$ for all $x \in \Omega$. To that end, we apply Lemma 4.1 taking $w(t, x)=1-u(t, x)$; since

$$
\left.\frac{\partial}{\partial r}\right|_{r=0} \int_{\Omega(r)}(1-u(t, x)) \mathrm{d} x=-\operatorname{vol}(\partial \Omega)
$$

for all $t$, we obtain:

$$
\begin{aligned}
H(t)=\operatorname{vol}(\Omega) & -\frac{2}{\sqrt{\pi}} \operatorname{vol}(\partial \Omega) \sqrt{t} \\
& +\int_{0}^{t} \int_{0}^{\infty} e(t-\tau, r, 0) \rho_{*}((1-u(\tau, \cdot)) \Delta \rho)(r) \mathrm{d} r \mathrm{~d} \tau
\end{aligned}
$$

We use (11) to obtain the following theorem:

THEOREM 4.2. - If $\Omega$ is a convex polyhedron in the $n$-dimensional euclidean space, then

$$
\int_{\Omega} u(t, x) \mathrm{d} x=\operatorname{vol}(\Omega)-\frac{2}{\sqrt{\pi}} \operatorname{vol}(\partial \Omega) \sqrt{t}+c_{2} t+\ell(t)
$$

with

$$
c_{2}=4 \sum_{E} \operatorname{vol}_{n-2}(E) \cdot \int_{0}^{\infty}\left(1-\frac{\tanh (\gamma(E) x)}{\tanh (\pi x)}\right) \mathrm{d} x
$$

TOME $129-2001-\mathrm{N}^{\mathrm{O}} 4$ 
and where $E$ runs through the family of all $(n-2)$-dimensional faces of $\Omega$ (the "edges" if $n=3$ ), and $\gamma(E)$ is the interior angle of the two $(n-1)$-planes whose intersection is $E$. The remainder $\ell(t)$ is bounded, in absolute value, for all $t$, by $C t^{3 / 2}+h(t)$ for a constant $C$, and for $a$ function $h(t)$ which is exponentially decreasing as $t \rightarrow 0(C$ and $h(t)$ are explicited in (26)).

Let us fix some notation. The closure $\bar{\Omega}$ of $\Omega$ is a polytope, in the sense that it is the intersection of a finite family $I=\{1, \ldots, m\}$ of closed half-spaces $\mathcal{H}_{i}$. We may write $\mathcal{H}_{i}=\left\{x \in \mathbb{R}^{n}: \rho_{\pi_{i}}(x) \geq 0\right\}$ where $\rho_{\pi_{i}}$ denotes the distance, taken with sign, from the oriented affine hyperplane $\pi_{i}$ of $\mathbb{R}^{n}$. Note that $\rho_{\pi_{i}}$ is an affine map. The $(n-1)$-dimensional faces of $\bar{\Omega}$ are the subsets of $\partial \Omega$ defined by $\mathcal{F}_{i}=\pi_{i} \cap \bar{\Omega}$ for $i \in I$. Each $\mathcal{F}_{i}$ is a polytope in $\pi_{i}$; the hyperplanes which bound $\mathcal{F}_{i}$ are given by all the intersections $\pi_{i} \cap \pi_{j}$, with $j \neq i$ (with the obvious orientation). In turn, each $(n-2)$-dimensional face $\mathcal{F}_{i} \cap \mathcal{F}_{j}$, with $j \neq i$, is a polytope in the $(n-2)$-dimensional euclidean space $\pi_{i} \cap \pi_{j}$, and so on. By $\operatorname{vol}_{d}(P)$ we denote the Lebesgue measure of the polytope $P$ in $\mathbb{R}^{d}$, and by $\gamma_{i j}$ we denote the interior angle at $\mathcal{F}_{i} \cap \mathcal{F}_{j}$ : it is the unique angle between 0 and $\pi$ such that $\cos \left(\gamma_{i j}\right)=-\nu_{i} \cdot \nu_{j}$, where $\nu_{i}$ and $\nu_{j}$ are the respective unit normal vectors of $\pi_{i}$ and $\pi_{j}$, positively oriented. Note that, if $\mathcal{F}_{i}$ and $\mathcal{F}_{j}$ are incident faces, then $0<\gamma_{i j}<\pi$.

Our aim is then to prove that

$$
c_{2}=2 \sum_{i \neq j} \operatorname{vol}_{n-2}\left(\mathcal{F}_{i} \cap \mathcal{F}_{j}\right) \cdot \int_{0}^{\infty}\left(1-\frac{\tanh \left(\gamma_{i j} x\right)}{\tanh (\pi x)}\right) \mathrm{d} x .
$$

The proof proceeds in the following way: we first describe the cut-locus of $\partial \Omega$, show that it is a polyhedral set (i.e. a finite union of polytopes) and give a convenient expression of $\Delta_{\text {Cut }} \rho$ as integration on the cut-locus. We then give the proof in four steps. Finally, we examine the special case $n=2$, and extend our proof to cover the (not necessarily convex) polygonal domains in the plane (Theorem 4.10), already obtained in [3].

4.1. Description of the cut-locus of a convex polyhedron. - Let $\rho$ : $\Omega \rightarrow \mathbb{R}$ denote the distance from $\partial \Omega$. We observe the following fact, which follows easily from the convexity of $\Omega$ : for all $x \in \Omega$,

$$
\rho(x)=\min _{i=1, \ldots, m} \rho_{\pi_{i}}(x) .
$$

Since there are no focal points of $\partial \Omega$, the cut-locus of $\partial \Omega$ is the closure of the set of points of $\Omega$ which can be joined to $\partial \Omega$ by at least two minimizing line segments. Therefore:

$$
\operatorname{Cut}(\partial \Omega)=\bigcup_{i \neq j} \operatorname{Cut}_{i j} \quad \text { with } \quad \operatorname{Cut}_{i j}=\left\{x \in \bar{\Omega}: \rho(x)=\rho_{\pi_{i}}(x)=\rho_{\pi_{j}}(x)\right\} .
$$


Proposition 4.3. - (i) For each $i \neq j$, Cut ${ }_{i j}$ is a polytope in the hyperplane $\pi_{i j}=\left\{x \in \Omega: \rho_{\pi_{i}}(x)=\rho_{\pi_{j}}(x)\right\}$ (the "bisecting hyperplane" of $\pi_{i}$ and $\pi_{j}$ );

(ii) Let $\phi \in C^{0}(\bar{\Omega})$, and $\psi \in C^{0}([0, \infty))$. Then:

$$
\begin{aligned}
\int_{\Omega} \phi \Delta \rho & =\sum_{i \neq j} \cos \left(\frac{\gamma_{i j}}{2}\right) \int_{\mathrm{Cut}_{i j}} \phi(x) \mathrm{d} x \quad \text { and } \\
\int_{0}^{\infty} \psi \rho_{*}(u \Delta \rho) & =\sum_{i \neq j} \cos \left(\frac{\gamma_{i j}}{2}\right) \int_{\mathrm{Cut}_{i j}} u(x) \psi(\rho(x)) \mathrm{d} x,
\end{aligned}
$$

$\mathrm{d} x$ denoting Lebesgue measure on the hyperplane $\pi_{i j}$ of $\mathbb{R}^{n}$.

Proof. - (i) Consider the set

$$
\overline{\mathcal{R}}_{i}=\left\{x \in \Omega: \rho(x)=\rho_{\pi_{i}}(x)\right\} .
$$

Then $\bar{\Omega}=\sum_{i \in I} \overline{\mathcal{R}}_{i}$; moreover, for each $j \neq i, \overline{\mathcal{R}}_{i}$ lies entirely on one side of the bisecting hyperplane $\pi_{i j}$ : denote by $\mathcal{H}_{i j}$ the closed half-space having that property. Then it is easy to show that $\overline{\mathcal{R}}_{i}=\bigcap_{j \in I} \mathcal{H}_{i j}$ (we agree to set $\left.\mathcal{H}_{i i}=\mathcal{H}_{i}\right)$. Hence $\overline{\mathcal{R}}_{i}$ is a polytope, and since $\mathrm{Cut}_{i j}$ is one of its faces, so is $\mathrm{Cut}_{i j}$. Note also that the faces of $\mathrm{Cut}_{i j}$ are $\mathcal{F}_{i} \cap \mathcal{F}_{j}$, together with all polytopes $\mathrm{Cut}_{i j k} \equiv \overline{\mathcal{R}}_{i} \cap \overline{\mathcal{R}}_{j} \cap \overline{\mathcal{R}}_{k}$ with $k \in I, k \neq i, k \neq j$.

Proof of (ii): we assume $\phi$ smooth; the assertion will follow by a density argument. As $\Delta \rho=0$ on the interior of $\overline{\mathcal{R}}_{i}$, by Green's formula and the fact that the boundary of $\overline{\mathcal{R}}_{i}$ consists of $\mathcal{F}_{i}$ together with all $\mathrm{Cut}_{i j}(j \neq i)$, we get:

$$
0=\int_{\overline{\mathcal{R}}_{i}} \phi \Delta \rho=\int_{\overline{\mathcal{R}}_{i}} \nabla \phi \cdot \nabla \rho+\int_{\mathcal{F}_{i}} \phi \mathrm{d} v_{n-1}+\sum_{j \neq i} \int_{\mathrm{Cut}_{i j}} \phi\left(\nabla \rho \cdot \nu_{i j}\right) \mathrm{d} v_{n-1},
$$

where $\nu_{i j}$ is the unit normal to $\pi_{i j}$ (oriented towards the interior of $\overline{\mathcal{R}}_{i}$ ). As $\cos \left(\frac{1}{2} \gamma_{i j}\right)=-\nabla \rho \cdot \nu_{i j}$, we get the first formula by summation and by Green's formula again. The second formula follows from the first by the definition of push-forward.

By the representation (11) of the heat content, and Proposition 4.3:

$$
\begin{aligned}
H(t) & -\operatorname{vol}(\Omega)+\frac{2}{\sqrt{\pi}} \operatorname{vol}(\partial \Omega) \sqrt{t} \\
& =\sum_{i \neq j} \cos \left(\frac{\gamma_{i j}}{2}\right) \int_{0}^{t} \int_{\mathrm{Cut}_{i j}} e(t-\tau, \rho(x), 0)(1-u(\tau, x)) \mathrm{d} x \mathrm{~d} \tau
\end{aligned}
$$

so $c_{2}$ is the coefficient of $t$ in the asymptotic expansion of the right-hand side of (12) as $t \rightarrow 0$. To compute it, we restrict to a suitable $\epsilon$-neighborhood of $\partial \Omega$. Let us fix some notation on the incidence relations of the $\mathcal{F}_{i}^{\prime} s$, and set:

$$
\begin{aligned}
& I_{2}=\left\{(i, j) \in I \times I: i \neq j, \mathcal{F}_{i} \cap \mathcal{F}_{j} \neq \varnothing\right\} ; \\
& I_{3}=\left\{(i, j, k) \in I \times I \times I: i \neq j \neq k \neq i, \mathcal{F}_{i} \cap \mathcal{F}_{j} \cap \mathcal{F}_{k} \neq \varnothing\right\} .
\end{aligned}
$$

TOME $129-2001-\mathrm{N}^{\mathrm{O}} 4$ 
Then:

Lemma 4.4. - Let $\epsilon=\inf _{(i, j, k) \notin I_{3}} \operatorname{dist}\left(\mathrm{Cut}_{i j}, \mathcal{F}_{k}\right)$. Then $\epsilon>0$, and:

(i) If $x \in \mathrm{Cut}_{i j}$ and $(i, j) \notin I_{2}$, then $\rho(x) \geq \epsilon$;

(ii) If $x \in \mathrm{Cut}_{i j k}$ and $(i, j, k) \notin I_{3}$, then $\rho(x) \geq \epsilon$.

Proof. - Recall that Cut $\mathrm{Cu}_{i j}$ and $\mathcal{F}_{k}$ are closed subsets of $\mathbb{R}^{n}$. To show that $\epsilon>0$, it is then enough to show that, if $(i, j, k) \notin I_{3}$, then $\operatorname{Cut}_{i j} \cap \mathcal{F}_{k}=\varnothing$. But this is clear, since $\mathrm{Cut}_{i j} \cap \mathcal{F}_{k} \subseteq \mathcal{F}_{i} \cap \mathcal{F}_{j} \cap \mathcal{F}_{k}$.

Proof of (ii): if $x \in \mathrm{Cut}_{i j k}$, then $\rho(x)=d(x, z)$ for some $z \in \mathcal{F}_{k}$. If $(i, j, k) \notin$ $I_{3}$, we have $d(x, z) \geq \epsilon$ by our definition of $\epsilon$, and (ii) is proved.

Proof of (i): let $(i, j) \notin I_{2}$; the restriction of $\rho$ to $\mathrm{Cut}_{i j}$ is just $\rho_{\pi_{i}}$ : an affine map. Hence $\left.\rho\right|_{\text {Cut }_{i j}}$ attains its absolute minimum on the boundary of Cut ${ }_{i j}$ : this implies, since $\partial \mathrm{Cut}_{i j} \cap \partial \Omega=\varnothing$, that there exists an index $k, k \neq i, k \neq j$, and a point $y \in \mathrm{Cut}_{i j k}$ (see the proof of Proposition 4.3) such that $\rho(x) \geq \rho(y)$ for all $x \in \mathrm{Cut}_{i j}$. Since $(i, j) \notin I_{2}$, a fortiori $(i, j, k) \notin I_{3}$, hence $\rho(y) \geq \epsilon$ by (ii).

4.2. The main steps of the proof. - The proof of the theorem is in four steps, which we outline below. Set, for brevity:

$$
Z_{i j}(u ; \tau)=\int_{\mathrm{Cut}_{i j}} e(t-\tau, \rho(x), 0)(1-u(\tau, x)) \cdot \mathrm{d} x
$$

STEP 1. - If $\mathcal{F}_{i} \cap \mathcal{F}_{j}=\varnothing$, then $\mathrm{Cut}_{i j}$ is at distance $\geq \epsilon$ from $\partial \Omega$. Hence each pair $(i, j) \notin I_{2}$ contributes to the sum in (12) with an exponentially decreasing term. Precisely, since $Z_{i j}(u ; \tau) \leq e(t-\tau, \epsilon, 0) \operatorname{vol}_{n-1}\left(\mathrm{Cut}_{i j}\right)$, and since, from Proposition 4.3 (ii) applied to $\phi=1$

$$
\sum_{(i, j) \notin I_{2}} \cos \left(\gamma_{i j} / 2\right) \operatorname{vol}_{n-1}\left(\mathrm{Cut}_{i j}\right) \leq \operatorname{vol}(\partial \Omega),
$$

we get

$$
\begin{gathered}
\left|H(t)-\operatorname{vol}(\Omega)+\frac{2}{\sqrt{\pi}} \operatorname{vol}(\partial \Omega) \sqrt{t}-\sum_{(i, j) \in I_{2}} \cos \left(\gamma_{i j} / 2\right) \int_{0}^{t} Z_{i j}(u ; \tau) \mathrm{d} \tau\right| \\
\leq \frac{4}{\sqrt{\pi} \epsilon^{2}} \operatorname{vol}(\partial \Omega) t^{3 / 2} \mathrm{e}^{-\epsilon^{2} / 4 t}
\end{gathered}
$$

In Steps $2-4$, we assume that $(i, j) \in I_{2}$ (that is, $\mathcal{F}_{i}$ and $\mathcal{F}_{j}$ are incident faces).

STEP 2. - It is the most delicate estimate. We show that, in order to compute the term in $t$ in the expansion of the heat content, we can replace the temperature function $u$ on $\mathrm{Cut}_{i j}$ by the temperature function $u_{i j}$, relative to 
the infinite open wedge $W_{i j}$ in $\mathbb{R}^{n}$ bounded by the oriented hyperplanes $\pi_{i}$ and $\pi_{j}$. Precisely:

$$
\left|\int_{0}^{t} Z_{i j}(u ; \tau) \mathrm{d} \tau-\int_{0}^{t} Z_{i j}\left(u_{i j} ; \tau\right) \mathrm{d} \tau\right| \leq C_{1}(i, j) t^{3 / 2}+C_{2}(i, j) t^{2} \mathrm{e}^{-\epsilon^{2} / 4 n t}
$$

for some positive constants $C_{1}(i, j), C_{2}(i, j)$ (see $\left.(23)\right)$. If $\operatorname{dim}(\Omega)=2$ then $C_{1}(i, j)=0$.

STEP 3. - We observe that, when restricted to $\pi_{i j}$ (the bisecting plane of the wedge $\left.W_{i j}\right)$, the temperature $u_{i j}(t, x)$ depends only on $\rho_{i j}(x)=$ distance of $x$ from $\pi_{i} \cap \pi_{j}$, so that it can be written $u_{i j}(t, x)=\tilde{u}_{i j}\left(t, \rho_{i j}(x)\right)$, where $\tilde{u}_{i j}$ is a function defined on $(0, \infty) \times[0, \infty)$. Hence we show that

$$
\begin{array}{r}
\left|\int_{0}^{t} Z_{i j}\left(u_{i j} ; \tau\right) \mathrm{d} \tau-\operatorname{vol}_{n-2}\left(\mathcal{F}_{i} \cap \mathcal{F}_{j}\right) \cdot c_{i j}(t)\right| \\
\leq C_{3}(i, j) t^{3 / 2}+C_{4}(i, j) t^{2} \mathrm{e}^{-\epsilon^{2} / 4 t},
\end{array}
$$

where $C_{3}(i, j), C_{4}(i, j)$ are positive constants (see $(25)$ ), and

$$
c_{i j}(t)=\int_{0}^{t} \int_{0}^{\infty} e\left(t-\tau, r \sin \left(\gamma_{i j} / 2\right), 0\right)\left(1-\tilde{u}_{i j}(\tau, r)\right) \mathrm{d} r \mathrm{~d} \tau .
$$

If $\operatorname{dim}(\Omega)=2$, then $C_{3}(i, j)=0$.

STEP 4. - It is the explicit computation:

$$
c_{i j}(t)=\frac{2}{\cos \left(\gamma_{i j} / 2\right)} \int_{0}^{\infty}\left(1-\frac{\tanh \left(\gamma_{i j} x\right)}{\tanh (\pi x)}\right) \mathrm{d} x \cdot t .
$$

The theorem follows from formulas (14) to (18). See (26) for the explicit expressions of $C$ and $h(t)$.

4.3. The proofs of Steps 1-4. - We make use, several times, of the following, easily established, facts:

F1. - If $S$ is a $p$-dimensional affine subspace of $\mathbb{R}^{n}$, then: $\operatorname{vol}_{p}(S \cap \Omega) \leq B_{p}$, where we have set $B_{p}=\operatorname{vol}\left(B^{p}(\operatorname{diam}(\Omega))\right)$ and where $B^{p}(a)$ is the ball of radius $a$ in $\mathbb{R}^{p}$. Then $\operatorname{vol}\left(B^{0}(a)\right)=1$, and we set $\operatorname{vol}\left(B^{p}(a)\right)=0$ if $p<0$.

F2. - If $\rho_{\pi}: \mathbb{R}^{n} \rightarrow \mathbb{R}$ is the distance function from the oriented hyperplane $\pi$, with unit normal $\nu=\nabla \rho$, then the gradient of the restriction of $\rho_{\pi}$ to the affine subspace $S$ of $\mathbb{R}^{n}$ is constant, and is equal to the orthogonal projection of $\nabla \rho_{\pi}$ onto $S$.

Proof of 15. - We now fix $(i, j) \in I_{2}$ and introduce the notation

$$
I_{i j}=\left\{k \in I:(i, j, k) \in I_{3}\right\} .
$$

TOME $129-2001-\mathrm{N}^{\mathrm{O}} 4$ 
Since $\mathcal{F}_{i}$ and $\mathcal{F}_{i}$ are incident, so are the hyperplanes $\pi_{i}$ and $\pi_{j}$. We denote by $W_{i j}$ the infinite open wedge in $\mathbb{R}^{n}$ given by the intersection of the two half-spaces determined by $\pi_{i}$ and $\pi_{j}$, i.e.

$$
W_{i j}=\left\{x: \rho_{\pi_{i}}(x)>0\right\} \cap\left\{x: \rho_{\pi_{j}}(x)>0\right\} .
$$

Note that $\Omega \subseteq W_{i j}$. We then let $u_{i j}:(0, \infty) \times W_{i j} \rightarrow \mathbb{R}$ denote the solution of the heat equation on $W_{i j}$, which satisfies the Dirichlet condition on the boundary of $W_{i j}$, and has unit initial conditions $u_{i j}(0, \cdot)=\mathbf{1}_{W_{i j}}$.

LemmA 4.5. - Let $A_{i j}=\bigcup_{k \neq i, k \neq j} \mathcal{F}_{k}$. Then, for all $t>0$ :

$$
\begin{aligned}
\left|\int_{0}^{t} Z_{i j}(u ; \tau) \mathrm{d} \tau-\int_{0}^{t} Z_{i j}\left(u_{i j} ; \tau\right) \mathrm{d} \tau\right| \\
\leq n \int_{0}^{t} \int_{\operatorname{Cut}_{i j}} e(t-\tau, \rho(x), 0) \mathrm{e}^{-d\left(x, A_{i j}\right)^{2} / 4 n t} \mathrm{~d} x \mathrm{~d} \tau
\end{aligned}
$$

Proof. - We introduce the function $v_{i j}(t, x)$, which is the solution of the heat equation on $\mathbb{R}^{n}$ satisfying the boundary conditions $v_{i j}(t, x) \equiv 1$ when $x \in A_{i j}$, and the initial conditions $v_{i j}(0, \cdot) \equiv 0$. From Levy's maximal inequality

$$
v_{i j}(t, x) \leq 2 \int_{\|y\| \geq d\left(x, A_{i j}\right)} \frac{e^{-\|y\|^{2} / 4 t}}{(4 \pi t)^{n / 2}} \mathrm{~d} y
$$

(for an equivalent, probabilistic formulation of this inequality, we refer to [18, Thm 3.6.5]). Now it is easy to verify that the restrictions of $u_{i j}-u$ and $v_{i j}$ to $\Omega$ are both solutions of the heat equation on $\Omega$; they have the same initial conditions on $\Omega$, and moreover, since $v_{i j} \geq 0$, and $0 \leq u_{i j}-u \leq 1$, we have

$$
\left.\left(u_{i j}-u\right)\right|_{\partial \Omega} \leq\left. v_{i j}\right|_{\partial \Omega}
$$

for all $t>0$. Therefore, for all $t>0$, and $x \in \Omega$ :

$$
0 \leq u_{i j}(t, x)-u(t, x) \leq v_{i j}(t, x) .
$$

It remains to estimate the integral in the right-hand side of (19). Let

$$
I_{n}(b)=\int_{\|y\| \geq b} \frac{\mathrm{e}^{-\|y\|^{2} / 4 t}}{(4 \pi t)^{n / 2}} \mathrm{~d} y, \quad J(b)=\int_{-b}^{b} \frac{\mathrm{e}^{-r^{2} / 4 t}}{(4 \pi t)^{1 / 2}} \mathrm{~d} r .
$$

As $(-b / \sqrt{n}, b / \sqrt{n})^{n} \subseteq B^{n}(0 ; b) \subseteq(-b, b)^{n}$, we have

$$
J\left(\frac{b}{\sqrt{n}}\right)^{n} \leq 1-I_{n}(b) \leq J(b)^{n},
$$

and thus $1-I_{n}(b) \geq\left(1-I_{2}(b / \sqrt{n})\right)^{n / 2}$. Using polar coordinates, one finds $I_{2}(b)=\mathrm{e}^{-b^{2} / 4 t}$, and then we get $I_{n}(b) \leq \frac{1}{2} n \mathrm{e}^{-b^{2} / 4 t}$. The lemma follows by 
recalling the definition (13) of $Z_{i j}(u ; \tau)$. We finally observe the inequality (which will be used later):

$$
\int_{b}^{\infty} \frac{\mathrm{e}^{-r^{2} / 4 t}}{(\pi t)^{1 / 2}} \leq \mathrm{e}^{-b^{2} / 4 t}
$$

which holds since $\mathrm{e}^{-b^{2} / 4 t}=I_{2}(b) \geq 1-J(b)^{2} \geq 1-J(b)$.

REMARK 4.6. - If $\operatorname{dim}(\Omega)=2$, then (15) is an immediate consequence of Lemma 4.5: in fact, in that case $d\left(x, A_{i j}\right) \geq \epsilon$ for all $x \in \mathrm{Cut}_{i j}$, by our definition of $\epsilon$, and therefore the left-hand side of (15) will be exponentially decreasing as $t \rightarrow 0$, with $C_{1}(i, j)=0$, and

$$
C_{2}(i, j)=\frac{4}{\epsilon^{2} \sin \left(\gamma_{i j} / 2\right)} t^{2} \mathrm{e}^{-\epsilon^{2} / 4 t} .
$$

If $\operatorname{dim}(\Omega)>2$, then $A_{i j}$ will intersect $\mathrm{Cut}_{i j}$ in the set $\bigcup_{k \neq i, k \neq j}\left(\mathcal{F}_{i} \cap \mathcal{F}_{j} \cap \mathcal{F}_{k}\right)$ which is not empty, in general. Therefore we must proceed with the proof and show that

$$
n \int_{0}^{t} \int_{\mathrm{Cut}_{i j}} e(t-\tau, \rho(x), 0) \mathrm{e}^{-d\left(x, A_{i j}\right)^{2} / 4 n \tau} \mathrm{d} x \mathrm{~d} \tau
$$

is indeed $0\left(t^{3 / 2}\right)$, as $t \rightarrow 0$.

For $x \in \pi_{i j}$, let $\rho_{i j}(x)$ stand for the distance of $x$ from the hyperplane $\pi_{i} \cap \pi_{j}$ of $\pi_{i j}$. Observe that, if $x \in \mathrm{Cut}_{i j}$, then $\rho(x)=\rho_{i j}(x) \sin \left(\frac{1}{2} \gamma_{i j}\right)$. Hence, by the co-area formula, applied to $\rho_{i j}: \mathrm{Cut}_{i j} \rightarrow \mathbb{R}$ :

$$
\begin{aligned}
& \int_{\mathrm{Cut}_{i j}} e(t-\tau, \rho(x), 0) \mathrm{e}^{-d\left(x, A_{i j}\right)^{2} / 4 n \tau} \mathrm{d} x \\
& \quad=\int_{0}^{\infty} e\left(t-\tau, r \sin \left(\gamma_{i j} / 2\right), 0\right) \int_{\rho_{i j}^{-1}(r) \cap \mathrm{Cut}_{i j}} \mathrm{e}^{-d\left(x, A_{i j}\right)^{2} / 4 n \tau} \mathrm{d} x \mathrm{~d} r .
\end{aligned}
$$

Next, since $d\left(x, A_{i j}\right)=\min _{k \neq i, k \neq j} d\left(x, \mathcal{F}_{k}\right)$ :

$$
\int_{\rho_{i j}^{-1}(r) \cap \mathrm{Cut}_{i j}} \mathrm{e}^{-d\left(x, A_{i j}\right)^{2} / 4 n \tau} \mathrm{d} x \leq \sum_{k \neq i, k \neq j} \int_{\rho_{i j}^{-1}(r) \cap \mathrm{Cut}_{i j}} \mathrm{e}^{-d\left(x, \mathcal{F}_{k}\right)^{2} / 4 n \tau} \mathrm{d} x .
$$

For a fixed $r, \rho_{i j}^{-1}(r) \cap \mathrm{Cut}_{i j}$ is contained in an $(n-2)$-hyperplane section of $\Omega$; hence, by our definition of $\epsilon$, we see that each term of the above sum involving an index $k \notin I_{i j}$ (that is, an index such that $\left.(i, j, k) \notin I_{3}\right)$ is majorized by $B_{n-2} \mathrm{e}^{-\epsilon^{2} / 4 n \tau}$.

Hence it remains to examine the integrals of type

where $k \in I_{i j}$.

$$
\int_{\rho_{i j}^{-1}(r) \cap \mathrm{Cut}_{i j}} \mathrm{e}^{-d\left(x, \mathcal{F}_{k}\right)^{2} / 4 n \tau} \mathrm{d} x
$$

TOME $129-2001-\mathrm{N}^{\mathrm{O}} 4$ 
First, note that $d\left(x, \mathcal{F}_{k}\right) \geq \rho_{\pi_{k}}(x)$. Now fix $r \geq 0$, and consider the $(n-2)$ dim polyhedron

$$
Q_{i j}=\rho_{i j}^{-1}(r) \cap \mathrm{Cut}_{i j}
$$

which lies in a hyperplane parallel to $\pi_{i} \cap \pi_{j}$. The function $\rho_{\pi_{k}}$, when restricted to $Q_{i j}$, has gradient (by property F2) given by

$$
P_{i j k}=\text { orthogonal projection of } \nabla \rho_{\pi_{k}} \text { onto } \pi_{i} \cap \pi_{j},
$$

and $\left\|P_{i j k}\right\|>0$ since, by assumption, $\mathcal{F}_{i} \cap \mathcal{F}_{j} \cap \mathcal{F}_{k} \neq \varnothing$, and so $\pi_{k}$ is incident $\pi_{i} \cap \pi_{j}$. By the co-area formula, applied to $\rho_{\pi_{k}}: Q_{i j} \rightarrow \mathbb{R}$, and by property F1:

$$
\begin{aligned}
\int_{Q_{i j}} \mathrm{e}^{-d\left(x, \mathcal{F}_{k}\right)^{2} / 4 n \tau} \mathrm{d} x & \leq \frac{1}{\left\|P_{i j k}\right\|} \int_{0}^{\infty} \mathrm{e}^{-s^{2} / 4 n \tau} \cdot \operatorname{vol}_{n-3}\left(\rho_{\pi_{k}}^{-1}(s) \cap Q_{i j}\right) \mathrm{d} s \\
& \leq \frac{\sqrt{n \pi} B_{n-3}}{\left\|P_{i j k}\right\|} \tau^{1 / 2} .
\end{aligned}
$$

Summing over $k \neq i, k \neq j$, and taking into account formulas (20), (21) and (22), we obtain:

$$
\begin{array}{rl}
\int_{\mathrm{Cut}_{i j}} & e(t-\tau, \rho(x), 0) \mathrm{e}^{-d\left(x, A_{i j}\right)^{2} / 4 n \tau} \mathrm{d} x \\
\leq & \frac{(m-2) B_{n-2}}{\sin \left(\gamma_{i j} / 2\right)} \mathrm{e}^{-\epsilon^{2} / 4 n \tau}+\left(\frac{\sqrt{n \pi} B_{n-3}}{\sin \left(\gamma_{i j} / 2\right)} \cdot \sum_{k \in I_{i j}} \frac{1}{\left\|P_{i j k}\right\|}\right) \cdot \tau^{1 / 2} .
\end{array}
$$

Integrating the above inequality from $\tau=0$ to $\tau=t$, multiplying by $n$, and applying Lemma 4.5, we obtain (15) with:

$$
\left\{\begin{array}{l}
C_{1}(i, j)=\frac{2 n \sqrt{n \pi} B_{n-3}}{3 \sin \left(\gamma_{i j} / 2\right)} \sum_{k \in I_{i j}} \frac{1}{\left\|P_{i j k}\right\|}, \\
C_{2}(i, j)=\frac{4 n^{2}(m-2) B_{n-2}}{\epsilon^{2} \sin \left(\gamma_{i j} / 2\right)}
\end{array}\right.
$$

Proof of (16). - We have already observed that, when restricted to the bisecting plane $\pi_{i j}$ of $\pi_{i}$ and $\pi_{j}$, the function $u_{i j}(\tau, x)$ depends only on the distance $\rho_{i j}(x)$ of $x$ from $\pi_{i} \cap \pi_{j}$; so let us set $u_{i j}(\tau, x)=\tilde{u}_{i j}\left(\tau, \rho_{i j}(x)\right)$ with $\tilde{u}_{i j}$ depending on $\tau$ and $r \geq 0$. By the co-area formula, applied to the map $\rho_{i j}: \mathrm{Cut}_{i j} \rightarrow \mathbb{R}$, and the definition (17) of $c_{i j}(t)$, writing for brevity

$$
V_{i j}(r)=\operatorname{vol}_{n-2}\left(\rho_{i j}^{-1}(r) \cap \mathrm{Cut}_{i j}\right)
$$

we have

$$
\begin{aligned}
& (24)\left|\int_{0}^{t} \int_{\operatorname{Cut}_{i j}} e(t-\tau, \rho(x), 0)\left(1-u_{i j}(\tau, x)\right) \mathrm{d} x \mathrm{~d} \tau-\operatorname{vol}_{n-2}\left(\mathcal{F}_{i} \cap \mathcal{F}_{j}\right) \cdot c_{i j}(t)\right| \\
& \quad \leq \int_{0}^{t} \int_{0}^{\infty} e\left(t-\tau, r \sin \left(\gamma_{i j} / 2\right), 0\right)\left(1-\tilde{u}_{i j}(\tau, r)\right)\left|V_{i j}(r)-V_{i j}(0)\right| \mathrm{d} r \mathrm{~d} \tau
\end{aligned}
$$


Lemma 4.7. - For $0<r<\epsilon / \sin \left(\frac{1}{2} \gamma_{i j}\right)$ one has

$$
\left|V_{i j}(r)-V_{i j}(0)\right| \leq B_{n-3}\left|\sum_{k \in I_{i j}} \cot \gamma_{i j k}\right| \cdot r,
$$

where $\gamma_{i j k}$ is the angle between the faces $\mathcal{F}_{i} \cap \mathcal{F}_{j}$ and $\mathrm{Cut}_{i j k}$ of the polytope $\mathrm{Cut}_{i j}$.

Proof. - See Appendix C.

We now split at $r=\epsilon / \sin \left(\frac{1}{2} \gamma_{i j}\right)$ the inner integral in the right-hand side of (24). By Lemma 4.7, and the fact that $\int_{\epsilon}^{\infty} e(t-\tau, s, 0) \mathrm{d} s \leq \mathrm{e}^{-\epsilon^{2} / 4(t-\tau)}$ (see the proof of Lemma 4.5), we have (16) with

$$
\left\{\begin{array}{l}
C_{3}(i, j)=\frac{4 B_{n-3}}{3 \sqrt{\pi} \sin ^{2}\left(\frac{1}{2} \gamma_{i j}\right)}\left|\sum_{k \in I_{i j}} \cot \gamma_{i j k}\right|, \\
C_{4}(i, j)=\frac{4 B_{n-2}}{\epsilon^{2} \sin \left(\frac{1}{2} \gamma_{i j}\right)} .
\end{array}\right.
$$

REMARK 4.8. - If $\operatorname{dim}(\Omega)=2$, then $V_{i j}(r)-V_{i j}(0)=0$ for $0<r<$ $\epsilon / \sin \left(\frac{1}{2} \gamma_{i j}\right)$, and therefore we see that in that case $C_{3}(i, j)=0$, and $C_{4}(i, j)=\frac{4}{\epsilon^{2} \sin \left(\frac{1}{2} \gamma_{i j}\right)} t^{2} \mathrm{e}^{-\epsilon^{2} / 4 t}$.

Proof of (18). - To compute $c_{i j}(t)$, we first take its Laplace transform. Then:

$$
C_{i j}(s) \stackrel{\text { def }}{=} \int_{0}^{\infty} c_{i j}(t) \mathrm{e}^{-s t} \mathrm{~d} t=s^{-1 / 2} \int_{0}^{\infty} \mathrm{e}^{-\sqrt{s} r \sin \left(\gamma_{i j} / 2\right)}\left(\frac{1}{s}-\tilde{U}_{i j}(s, r)\right) \mathrm{d} r,
$$

where $\tilde{U}_{i j}(\cdot, r)$ is the Laplace transform, with respect to time $t$, of $\tilde{u}_{i j}(\cdot, r)$. We will write down an explicit expression of $\frac{1}{s}-\tilde{U}_{i j}(s, r)$. First observe that $W_{i j}$ is isometric with $W\left(\gamma_{i j}\right) \times \mathbb{R}^{n-2}$ (with the product metric), if we denote by $W\left(\gamma_{i j}\right)$ the open wedge in $\mathbb{R}^{2}$ with interior angle $\gamma_{i j}$. We adopt cylindrical coordinates $x=(r, \alpha, y)$ where $(r, \alpha)$ are polar coordinates in $W\left(\gamma_{i j}\right)$ (the angle $\alpha$ being counted from the bisectrix of $\left.\gamma_{i j}\right)$, and where $y \in \mathbb{R}^{n-2}$. In these coordinates the temperature function $u_{i j}(t, x)$ is independent from $y$, hence it can be written, by a slight abuse of language, as $u_{i j}(t, r, \alpha)$. Note that $\tilde{u}_{i j}(t, r)=u_{i j}(t, r, 0)$.

The following lemma was suggested by the expression of the Green function of an open wedge in $\mathbb{R}^{2}$ as a Kontorovich-Lebedev transform (which we learned from [3]).

LEMMA 4.9. - Let $W(\gamma)$ be the open wedge in $\mathbb{R}^{2}$ with interior angle $\gamma$, and let $(r, \alpha)$ be polar coordinates with $\alpha \in\left(-\frac{1}{2} \gamma, \frac{1}{2} \gamma\right)$ being counted from the bisectrix

TOME $129-2001-\mathrm{N}^{\mathrm{O}} 4$ 
of $\gamma$. Let $u(t, r, \alpha)$ be the solution of:

$$
\left\{\begin{array}{l}
(\Delta+\partial / \partial t) u=0, \\
u(0, r, \alpha)=1, \quad r>0, \quad \alpha \in\left(-\frac{1}{2} \gamma, \frac{1}{2} \gamma\right), \\
u\left(t, r, \pm \frac{1}{2} \gamma\right)=0, \quad t>0, \quad r>0
\end{array}\right.
$$

and let $U(s, r, \alpha)=\int_{0}^{\infty} u(t, r, \alpha) \mathrm{e}^{-s t} \mathrm{~d} t$. Then:

$$
U(s, r, \alpha)=\frac{1}{s}-\frac{2}{\pi s} \int_{0}^{\infty} K_{i x}(\sqrt{s} r) \frac{\cosh \left(\frac{1}{2} \pi x\right) \cosh (\alpha x)}{\cosh \left(\frac{1}{2} \gamma x\right)} \mathrm{d} x,
$$

where $K_{i x}$ is the modified Bessel function of imaginary argument (see [11, 8.407.1]).

Proof. - In polar coordinates

$$
-\Delta=\frac{\partial^{2}}{\partial r^{2}}+\frac{1}{r} \frac{\partial}{\partial r}+\frac{1}{r^{2}} \frac{\partial^{2}}{\partial \alpha^{2}}
$$

Hence the function $U(s, r, \alpha)$ must satisfy, on the open wedge $W(\gamma)$, the boundary-value problem: $\Delta U=1-s U$, and $U\left(s, r, \pm \frac{1}{2} \gamma\right)=0$ for all $s>0$, and $r>0$. Now

$$
V(s, r, \alpha)=\frac{1}{s}-\frac{2}{\pi s} \int_{0}^{\infty} K_{i x}(\sqrt{s} r) \frac{\cosh \left(\frac{1}{2} \pi x\right) \cosh (\alpha x)}{\cosh \left(\frac{1}{2} \gamma x\right)} \mathrm{d} x
$$

is indeed a solution of $\Delta U=1-s U$ by [11, formula 8.491.6], and satisfies the given boundary conditions by [11, formula 6.794 .2$]$. The lemma follows.

We now come to the computation of $C_{i j}(s)$. From Lemma 4.9,

$$
\tilde{U}_{i j}(s, r)=U_{i j}(s, r, 0)=\frac{1}{s}-\frac{2}{\pi s} \int_{0}^{\infty} K_{i x}(\sqrt{s} r) \frac{\cosh \left(\frac{1}{2} \pi x\right)}{\cosh \left(\frac{1}{2} \gamma_{i j} x\right)} \mathrm{d} x,
$$

and therefore

$$
C_{i j}(s)=\frac{2}{\pi s^{3 / 2}} \int_{0}^{\infty} \frac{\cosh \left(\frac{1}{2} \pi x\right)}{\cosh \left(\frac{1}{2} \gamma_{i j} x\right)}\left(\int_{0}^{\infty} \mathrm{e}^{-\sqrt{s} r \sin \left(\frac{1}{2} \gamma_{i j}\right)} K_{i x}(\sqrt{s} r) \mathrm{d} r\right) \mathrm{d} x .
$$

The inner integral, after the substitution $z=\sqrt{s} r$, will become:

$$
\frac{\pi}{2 \cos \left(\frac{1}{2} \gamma_{i j}\right) s^{1 / 2}}\left(\frac{\cosh \left(\frac{1}{2} \gamma_{i j} x\right)}{\cosh \left(\frac{1}{2} \pi x\right)}-\frac{\sinh \left(\frac{1}{2} \gamma_{i j} x\right)}{\sinh \left(\frac{1}{2} \pi x\right)}\right),
$$

by [11] (formula 6.611.3). Substituting, and changing $\frac{1}{2} x$ to $x$, we then obtain:

$$
C_{i j}(s)=\frac{2}{\cos \left(\frac{1}{2} \gamma_{i j}\right)} \cdot \int_{0}^{\infty}\left(1-\frac{\tanh \left(\gamma_{i j} x\right)}{\tanh (\pi x)}\right) \mathrm{d} x \cdot \frac{1}{s^{2}} .
$$

Taking inverse Laplace transform, we obtain (18).

BULLETIN DE LA SOCIÉtÉ MATHÉMATIQUE DE FRANCE 
4.4. The remainder term. - Collecting the constants in (23) and (25), we obtain:

$$
\begin{gathered}
C=\frac{2 n \sqrt{n \pi} B_{n-3}}{3} \sum_{(i, j, k) \in I_{3}} \frac{\cot \left(\gamma_{i j} / 2\right)}{\left\|P_{i j k}\right\|} \\
+\frac{4 B_{n-3}}{3 \sqrt{\pi}} \sum_{(i, j, k) \in I_{3}} \frac{\left|\cot \left(\gamma_{i j k}\right)\right|}{\cos \left(\gamma_{i j} / 2\right)} \sin ^{2}\left(\gamma_{i j} / 2\right) ; \\
h(t)=\alpha_{1} t^{3 / 2} \mathrm{e}^{-\epsilon^{2} / 4 t}+\alpha_{2} t^{2} \mathrm{e}^{-\epsilon^{2} / 4 t}+\alpha_{3} t^{2} \mathrm{e}^{-\epsilon^{2} / 4 n t},
\end{gathered}
$$

with $P_{i j k}$ and $\gamma_{i j k}$ as in the proof of (15) and (16), respectively; and with

$$
\begin{gathered}
\alpha_{1}=\frac{4}{\sqrt{\pi} \epsilon^{2}} \operatorname{vol}(\partial \Omega), \quad \alpha_{2}=\frac{4 B_{n-2}}{\epsilon^{2}} \sum_{(i, j) \in I_{2}} \cot \left(\gamma_{i j} / 2\right), \\
\alpha_{3}=\frac{4 n^{2}(m-2) B_{n-2}}{\epsilon^{2}} \sum_{(i, j) \in I_{2}} \cot \left(\gamma_{i j} / 2\right) .
\end{gathered}
$$

If $\operatorname{dim}(\Omega)=2$, then $C=0$ and, looking back at the proofs of Steps $1-4$, $h(t)$ can be reduced to the following form:

$$
h(t)=\frac{4}{\sqrt{\pi} \epsilon^{2}} \operatorname{vol}(\partial \Omega) t^{3 / 2} \mathrm{e}^{-\epsilon^{2} / 4 t}+\frac{8}{\epsilon^{2}} \sum_{(i, j) \in I_{2}} \cot \left(\gamma_{i j} / 2\right) t^{2} \mathrm{e}^{-\epsilon^{2} / 4 t} .
$$

With this, the proof of Theorem 4.2 is complete.

\subsection{Heat content asymptotics of a polygonal domain in the plane.} - We now apply our methods to prove the two-dimensional, polygonal case.

Theorem 4.10 (van den Berg, Srisatkunarajah [3]). — Let $\Omega$ be a (not necessarily convex) polygonal domain in $\mathbb{R}^{2}$. Then:

$$
\int_{\Omega} u(t, x) \mathrm{d} x=A-\frac{2 L}{\sqrt{\pi}} \sqrt{t}+4 \sum_{P} c_{P} \cdot t+\ell(t)
$$

where $A$ is the area, $L$ is the length of the boundary. The sum is taken over all vertices $P$ of $\Omega$, and $c_{P}=\int_{0}^{\infty}(1-\tanh (\gamma x) / \tanh (\pi x)) \mathrm{d} x$, with $\gamma \in(0,2 \pi)$ denoting the interior angle at the vertex $P$. The remainder $\ell(t)$ is exponentially decreasing as $t \rightarrow 0$.

Proof. - If $\Omega$ is not convex, it is no longer true that the cut-locus is a polygonal set. However, we just need to describe the cut-locus and to control the measure $\Delta \rho$ in a small strip $\Omega_{\epsilon}=\{x \in \Omega: \rho(x)<\epsilon\}$ around the boundary: in fact, by (11), it is enough to study the double integral:

$$
\int_{0}^{t} \int_{0}^{\epsilon} \mathrm{e}(t-\tau, r, 0) \rho_{*}((1-u(\tau, \cdot)) \Delta \rho)(r) \mathrm{d} r \mathrm{~d} \tau
$$

TOME $129-2001-\mathrm{N}^{\mathrm{O}} 4$ 
and show that, as $t \rightarrow 0$, its behavior is of type $4 \sum_{P} c_{P} \cdot t+$ exponentially decreasing terms.

Now $\Delta \rho$ is zero near the edges and far from the vertices of the polygon; let us choose $\epsilon>0$ small in such a way that near a convex vertex $P($ where $\gamma \in(0, \pi)$ ) the cut-locus meets the strip $\Omega_{\epsilon}$ in a segment of the bisecting line $\operatorname{Cut}_{P}$ of the two sides meeting at $P$, and near a concave vertex $Q$ (where $\gamma \in(\pi, 2 \pi)$ ), the circular sector $B(Q ; \epsilon) \cap \Omega$ does not meet the cut-locus at all.

The contribution of a convex vertex $P$ to the double integral (27) is therefore:

$$
2 \cos (\gamma / 2) \int_{0}^{t} \int_{\mathrm{Cut}_{P} \cap \Omega_{\epsilon}} e(t-\tau, \rho(x), 0)(1-u(\tau, x)) \mathrm{d} x \mathrm{~d} \tau .
$$

We already proved that $(28)$ is equal to $4 c_{P} \cdot t+$ exponentially decreasing terms. Near a concave vertex $Q$, we have $\Delta_{\text {Cut }} \rho=0$, and the level curves of the distance function are $C^{1}$ curves given by the union of the two segments parallel to the two sides meeting at $Q$, and an arc of circle of angle $\gamma-\pi$. Precisely, in polar coordinates $(r, \alpha)$ centered at $Q$, with the angle $\alpha$ being counted from the bisectrix of $\gamma$, we have, for $0<r<\epsilon$ :

$$
\Delta_{\mathrm{reg}} \rho(r, \alpha)=\left\{\begin{array}{cl}
-r^{-1} & \text { if }-\frac{1}{2}(\gamma-\pi)<\alpha<\frac{1}{2}(\gamma-\pi) . \\
0 & \text { otherwise. }
\end{array}\right.
$$

On the circular sector $B(Q ; \epsilon) \cap \Omega_{\epsilon}$ we replace $u(t, x)$ by $u_{Q}(t, x)$, the temperature function on the infinite open wedge with vertex in $Q$ and interior angle $\gamma$; by Levy's maximal inequality, the error due to this approximation will be exponentially decreasing as $t \rightarrow 0$ (proceed as in Lemma 4.5). Recalling that $\rho_{*}$ is in this case integration on the level curves of the distance function, we conclude that the contribution of the concave vertex $Q$ to $(27)$ is, modulo exponentially decresing terms, given by:

$$
\int_{0}^{t} \int_{0}^{\infty} \mathrm{e}(t-\tau, r, 0) \int_{-(\gamma-\pi) / 2}^{(\gamma-\pi) / 2}\left(1-u_{Q}(\tau, r, \alpha)\right) \mathrm{d} \alpha \mathrm{d} r \mathrm{~d} \tau
$$

Its Laplace transform with respect to time $t$, at $s>0$, is, thanks to Lemma 4.9:

$$
-\frac{2}{\pi s^{3 / 2}} \int_{0}^{\infty} \mathrm{e}^{-\sqrt{s} r} \int_{-(\gamma-\pi) / 2}^{(\gamma-\pi) / 2} \int_{0}^{\infty} K_{i x}(\sqrt{s} r) \frac{\cosh \left(\frac{1}{2} \pi x\right) \cosh (\alpha x)}{\cosh \left(\frac{1}{2} \gamma x\right)} \mathrm{d} x \mathrm{~d} \alpha \mathrm{d} r,
$$

which can be evaluated again by [11], formula 6.611.3. One finds its value to be $\left(4 / s^{2}\right) c_{Q}$. Taking inverse Laplace transform, we obtain, also in this case, the vertex contribution $4 c_{Q} \cdot t$.

The remainder term of the asymptotic expansion of the heat content will be an exponentially decreasing function of $t$, as $t \rightarrow 0$, which depends on $\epsilon$, on $\operatorname{vol}(\partial \Omega)$, and on the angles $\gamma$; it can be easily estimated by the same methods used in Theorem 4.2. We omit the details.

BULLETIN DE LA SOCIÉtÉ MATHÉMATIQUE DE FRANCE 


\section{Appendix A}

LEMMA. - On any Riemannian manifold $M$, if $\rho$ is the distance function to a submanifold $N$, one has that $\Delta_{\mathrm{reg}} \rho \in L_{\mathrm{loc}}^{1}(M)$.

Proof. - We have to show that, if $K \subseteq M$ is compact, then $\int_{K}\left|\Delta_{\text {reg }} \rho\right|$ is finite. Let $R$ be large enough so that $K \subseteq \rho^{-1}[0, R)$. Then, integrating in normal coordinates:

$$
\int_{K}\left|\Delta_{\mathrm{reg}} \rho\right| \leq \int_{U(N)} \int_{0}^{\min \{c(\xi), R\}}\left|\theta^{\prime}(r, \xi)\right| \mathrm{d} r \mathrm{~d} \xi .
$$

Hence it is enough to show that $\left|\theta^{\prime}\right|$ is bounded on

$$
\{(r, \xi): 0<r<\min \{c(\xi), R\}, \xi \in U(N)\} .
$$

Let us consider the map $\Phi:(0, \infty) \times U(N) \rightarrow M$ defined by

$$
\Phi(r, \xi)=\exp _{\pi(\xi)} r \xi
$$

As $\Phi$ is everywhere $C^{\infty}$, its Jacobian determinant $\theta(r, \xi)=\Phi^{*}\left(\mathrm{~d} v_{n}\right) / \mathrm{d} r \wedge \mathrm{d} \xi$ (where $\mathrm{d} v_{n}$ and $\mathrm{d} \xi$ are the canonical volume forms of $M$ and $U(N)$, respectively) is also everywhere $C^{\infty}$. Now:

$$
\lim _{r \rightarrow 0} \theta^{\prime}(r, \xi)=\left\{\begin{array}{lll}
0 & \text { if } & \operatorname{dim}(N) \leq n-3, \\
1 & \text { if } & \operatorname{dim}(N)=n-2, \\
-\sum_{1 \leq i \leq n-1} \eta_{i}(\xi) & \text { if } & \operatorname{dim}(N)=n-1,
\end{array}\right.
$$

where $\eta_{i}(\xi)$ is the $i$-th principal curvature of $N$ at the unit normal vector $\xi$. From these facts, we deduce that $\left|\theta^{\prime}(r, \xi)\right|$ is indeed locally bounded on $(0, \infty) \times$ $U(N)$, and then that $\int_{K}\left|\Delta_{\text {reg }} \rho\right| \mathrm{d} v_{n}$ is finite.

The comparison theorems of Rauch and R.L. Bishop may be used to produce upper and lower bounds of $\theta^{\prime}(r, \xi)$ in terms of lower and upper bounds of the sectional (or Ricci) curvatures of $M$. We observe, in particular, that if $N$ is a $p$-dimensional submanifold of $\mathbb{R}^{n}$, then

$$
\theta(r, \xi)=r^{n-p-1} \prod_{i=1}^{p}\left(1-r \eta_{i}(\xi)\right)
$$

\section{Appendix B}

The scope of this appendix is to prove Lemma 2.2. We refer to $[5, \S 13.2]$ for the definition of the Hausdorff measures we use here.

TOME $129-2001-\mathrm{N}^{\mathrm{O}} 4$ 
Proof of Lemma 2.2. - By the definition of Hausdorff measure and our assumptions, we can find, for each $\epsilon>0$, a finite or countable covering of $K$ by sets $E_{i}(\epsilon), i=1,2, \ldots$, each of diameter not exceeding $\epsilon$, and satisfying:

$$
\sum_{i}\left(\operatorname{diam} E_{i}(\epsilon)\right)^{n-1} \leq \frac{2^{n-1}}{\operatorname{vol}\left(B^{n-1}\right)} \cdot H_{n-1}(K)+\epsilon,
$$

where $B^{n-1}$ is the open ball of radius 1 in $\mathbb{R}^{n-1}$. For each $i$ and each $\epsilon$, pick a point $x \in E_{i}(\epsilon) \cap K$; then the open ball $B_{i}(\epsilon)$ with center $x$ and radius $\delta \operatorname{diam} E_{i}(\epsilon)$, where $1<\delta<2$, contains $E_{i}(\epsilon)$. $K$ being compact, there is $k(\epsilon)$ such that $K \subseteq V(\epsilon) \stackrel{\text { def }}{=} \bigcup_{i=1}^{k(\epsilon)} B_{i}(\epsilon)$. Note that $V(\epsilon)$ is contained in a $2 \epsilon$-neighborhood of $K$, and that $\partial V(\epsilon)$ is piecewise smooth. Fix an open neighborhood $W$ of $K$, and let $\epsilon_{0}>0$ be a number such that $V(\epsilon) \subseteq W$ when $\epsilon<\epsilon_{0}$.

Claim. - Assume that Ricci $\geq-(n-1) \alpha^{2} g$ on $W$. Then there exists a positive constant $C_{1}$ depending only on $\alpha$ and $\epsilon_{0}$, such that

$$
\frac{\operatorname{vol}\left(\partial B_{i}(\epsilon)\right)}{\operatorname{radius}\left(B_{i}(\epsilon)\right)^{n-1}} \leq \operatorname{vol}\left(\partial B^{n-1}\right)+C_{1} \epsilon \quad \forall i=1, \ldots, k(\epsilon), \forall \epsilon<\epsilon_{0} .
$$

Proof of claim. - Using Bishop comparison theorem one argues that, if $B(x, r)$ is any ball contained in $W$, then $\operatorname{vol}(\partial B(x, r)) \leq \operatorname{vol}\left(\partial B_{-\alpha^{2}}(r)\right)$ where $B_{-\alpha^{2}}(r)$ is the ball of radius $r$ in the simply connected manifold of constant curvature $\sigma=-\alpha^{2}$. Hence it is enough to prove the claim in that case. Now a classical formula states that

$$
\operatorname{vol}\left(\partial B_{\sigma}(r)\right)=\operatorname{vol}\left(\partial B^{n-1}\right)\left(\frac{1}{\alpha} \sinh (\alpha r)\right)^{n-1} .
$$

Write $\sinh (\alpha r)=\alpha r\left(1+\psi_{\alpha}(r) r\right)$ with $\psi_{\alpha}(r)$ smooth and positive for $r \geq 0$ : the claim follows easily. Now:

$\operatorname{vol}(\partial V(\epsilon)) \leq \sum_{i=1}^{k(\epsilon)} \operatorname{vol}\left(\partial B_{i}(\epsilon)\right) \leq \delta^{n-1} \sum_{i=1}^{k(\epsilon)} \frac{\operatorname{vol}\left(\partial B_{i}(\epsilon)\right)}{\left(\operatorname{radius}\left(B_{i}(\epsilon)\right)\right)^{n-1}} \cdot\left(\operatorname{diam}\left(E_{i}(\epsilon)\right)\right)^{n-1}$

and we get the assertion by B.1, B.2 and the fact that $\delta$ was arbitrary. Proof is complete.

\section{Appendix C}

In this appendix we prove Lemma 4.7 , which is in fact a consequence of the following more general:

Lemma. - Let $P=$ polytope in $\mathbb{R}^{d}$ with faces $\mathcal{F}_{k}, k=1, \ldots, N ; \pi=$ hyperplane not intersecting the interior set of $P ; \gamma_{k}=\operatorname{angle}\left(\nu, \mathcal{F}_{k}\right)$, where $\nu$ is the unit normal to $\pi$, oriented inside $P ; \delta=\inf \operatorname{dist}\left(\mathcal{F}_{k}, \pi\right)$, where the infimum is taken over all indices $k$ such that $\mathcal{F}_{k}$ is not incident $\pi ; \rho_{\pi}: \mathbb{R}^{d} \rightarrow \mathbb{R}:$ distance 
from $\pi$. Then the map $V(r)=\operatorname{vol}_{d-1}\left(P \cap \rho_{\pi}^{-1}(r)\right)$ is differentiable on $(0, \delta)$ and in fact, for $0<r<\delta$ :

$$
V^{\prime}(r)=-\sum_{k=1}^{m} \cot \gamma_{k} \cdot \operatorname{vol}_{d-2}\left(\rho_{\pi}^{-1}(r) \cap \mathcal{F}_{k}\right),
$$

where $\mathcal{F}_{1}, \ldots, \mathcal{F}_{m}$ are the faces incident with $\pi$.

Proof. - For $0<r<\delta$ the hyperplane $\rho_{\pi}^{-1}(r)$ will intersect $\partial P$ only in the faces $\mathcal{F}_{1}, \ldots, \mathcal{F}_{m}$ and the section $\rho_{\pi}^{-1}(r) \cap P$ will be bounded by the $(d-2)$ dimensional faces $\mathcal{F}_{1} \cap \rho_{\pi}^{-1}(r), \ldots \mathcal{F}_{m} \cap \rho_{\pi}^{-1}(r)$. Consider the strip $P(r, h)=$ $P \cap\left\{r \leq \rho_{\pi} \leq r+h\right\}$. Since $\rho_{\pi}$ is an affine map, we have $\int_{P(r, h)} \Delta \rho_{\pi}=0$; now apply Green's theorem to the right-hand side, divide by $h$, and pass to the limit as $h \rightarrow 0$.

To prove Lemma 4.7, let $P=\mathrm{Cut}_{i j}$, and $\pi=\pi_{i} \cap \pi_{j}$ in the Lemma. Then $d=n-1, \rho_{\pi}=\rho_{i j}$ and $V(r)=\operatorname{vol}_{n-2}\left(\rho_{i j}^{-1}(r) \cap \mathrm{Cut}_{i j}\right)$. The faces of $P$ incident $\pi$ are then all polytopes $\mathrm{Cut}_{i j k}$ with $(i, j, k) \in I_{3}$. Moreover, if $x \in \mathrm{Cut}_{i j k}$, and $(i, j, k) \notin I_{3}$, then

$$
\rho_{i j}(x)=\frac{\rho(x)}{\sin \left(\frac{1}{2} \gamma_{i j}\right)} \geq \frac{\epsilon}{\sin \left(\frac{1}{2} \gamma_{i j}\right)}
$$

by our definition of $\epsilon$ (see Lemma 4.4 (ii)). Hence $\delta \geq \epsilon / \sin \left(\frac{1}{2} \gamma_{i j}\right)$ and Lemma 4.7 follows easily.

\section{Appendix D}

The scope of this appendix is to show that, in any Riemannian manifold, the cut-locus of a piecewise-smooth submanifold is a set of zero measure in the manifold. To achieve this result, we first define the "unit normal bundle" $\mathcal{U}(N)$ of $N$, and show that $\mathcal{U}(N)$ decomposes as a disjoint union of a regular part $\mathcal{U}_{\text {reg }}(N)$, and a singular part $\mathcal{U}_{\text {sing }}(N)$ which has zero measure in $\mathcal{U}(N)$. We can then carry out the classical procedure, valid when $N$ is smooth, with $\mathcal{U}_{\text {reg }}(N)$ replacing $U(N)$, and prove that the set of points where the distance function $\rho$ from $N$ is not smooth has zero measure in $M$ (Theorem D.1).

Let $N$ be a compact subset of a complete Riemannian manifold $M$. We say that $N$ is a piecewise-smooth submanifold of $M$ if $N$ is the disjoint union of a finite family $\mathcal{I}$ of smooth, open submanifolds $N_{i}$ of dimension $0 \leq n_{i} \leq n-1$. Let $\rho: M \rightarrow \mathbb{R}$ be the distance function from $N$. Then $\rho$ is Lipschitz. For each $i \in \mathcal{I}$, let $\mathcal{R}_{i}$ denote the maximal open subset of the set of all $x \in M$ for which there is a unique geodesic from $x$ to $N$ minimizing the distance from $N$, and the foot of this geodesic belongs to $N_{i}$.

Let $\mathcal{R}=\bigcup_{i} \mathcal{R}_{i}$. It is clear that, when restricted to $\mathcal{R}_{i}, \rho$ coincides with the smooth function $\rho_{N_{i}}=$ distance from $N_{i}$; moreover $\rho$ is $C^{\infty}$-smooth on $\mathcal{R}$, and

TOME $129-2001-\mathrm{N}^{\mathrm{O}} 4$ 
the regular Laplacian of $\rho$, defined on $\mathcal{R}$ by $\left.\Delta_{\text {reg }} \rho\right|_{\mathcal{R}_{i}}=\Delta\left(\left.\rho\right|_{\mathcal{R}_{i}}\right)$, satisfies

$$
\left.\Delta_{\mathrm{reg}} \rho\right|_{\mathcal{R}_{i}} \circ \Phi_{i}=-\frac{\theta_{N_{i}}^{\prime}}{\theta_{N_{i}}}
$$

where $\Phi_{i}$ is the normal chart relative to $N_{i}$, sending $(r, \xi) \in(0, \infty) \times U\left(N_{i}\right)$ to $\exp _{\pi(\xi)} r \xi \in M$, and $\theta_{N_{i}}$ is its Jacobian.

We now come to the main theorem of this appendix:

TheOREM D.1. - The complement of the open set $\mathcal{R}$ of all regular points of $\rho$ is of zero measure in $M$.

First, we define a surrogate of the "unit normal bundle" of $N, N$ being a compact subset of $M$. Let $U n(M)$ be the unit tangent bundle of $M$, and let $\pi: U n(M) \rightarrow M$ be its canonical projection. The cut-radius map: $c$ : $\pi^{-1}(N) \rightarrow[0, \infty]$ is defined in the usual way (see $\S 1$; no property of continuity is needed at this point). We set

$$
\mathcal{U}(N)=\left\{\xi \in \pi^{-1}(N): c(\xi)>0\right\}
$$

so that $\mathcal{U}(N)$ consists of all unit vectors which are based at points of $N$, and for which the corresponding geodesic minimizes the distance from $N$ on a segment of positive length. $\mathcal{U}(N)$ does indeed coincide with $U(N)$ when $N$ happens to be a smooth submanifold of $M$. The normal chart $\Phi:(0, \infty) \times \mathcal{U}(N) \rightarrow M$ where $\Phi(r, \xi)=\exp _{\pi(\xi)} r \xi$, is easily seen to be surjective on $M \backslash N$ and continuous.

Now assume that $N$ is a piecewise-smooth submanifold; then $U\left(N_{i}\right)$ is an open, smooth submanifold of $U n(M)$ of dimension $n-1$, having piecewisesmooth boundary. Set, for each $i$ in the index set $\mathcal{I}$ :

$$
\mathcal{U}\left(N_{i}\right)=\left\{\xi \in U\left(N_{i}\right): c(\xi)>0\right\} .
$$

Note that $\mathcal{U}(N)=\bigcup_{i} \mathcal{U}\left(N_{i}\right)$ since, if $\pi(\xi) \in N_{i}$ and $c(\xi)>0$, then $\xi$ must be normal to $N_{i}$. Now set $\mathcal{U}_{\text {reg }}(N)=\bigcup_{i} \mathcal{U}_{i}$, where $\mathcal{U}_{i}$ is the largest open subset of $U\left(N_{i}\right)$ contained in $\mathcal{U}\left(N_{i}\right)$. It follows that $\mathcal{U}_{\text {reg }}(N)$ is a smooth, open submanifold of $U n(M)$ of dimension $n-1$; it reduces to $U(N)$ if $N$ is smooth.

We will prove Theorem D.1 by applying the classical proof with $\mathcal{U}_{\text {reg }}(N)$ replacing $U(N)$. We first show that $\mathcal{U}(N) \backslash \mathcal{U}_{\text {reg }}(N)$ is, for our purposes, a negligible set.

Proposition D.2. - We have

$$
\mathcal{U}(N)=\mathcal{U}_{\text {reg }}(N) \cup \mathcal{U}_{\text {sing }}(N) \quad \text { (disjoint union) }
$$

and $\mathcal{U}_{\text {sing }}(N)$ is contained in a $(n-2)$-dimensional submanifold of $U n(M)$.

Proof. - We show that, in fact, $\mathcal{U}_{\text {sing }}(N) \subseteq \cup_{j}\left(\partial U\left(N_{j}\right)\right)$. Let $\xi \in \mathcal{U}_{\text {sing }}(N)$, say $\xi \in \mathcal{U}\left(N_{i}\right) \backslash \mathcal{U}_{\text {reg }}(N)$. If $\pi(\xi) \in \partial N_{i}$ we are done, since then $\xi \in \partial U\left(N_{i}\right)$. Hence assume $\pi(\xi) \in N_{i}$ : our aim is to show that then $\xi \in \partial U\left(N_{j}\right)$ for some $j \neq i$. Fix $r$ so that $0<r<c(\xi)$, and let $x=\Phi(r, \xi)$. The assumption $r<c(\xi)$ 
implies that $x$ can't be a focal point of $N_{i}$ along the geodesic $t \mapsto \Phi(t, \xi)$. Hence the normal map $\Phi=\Phi_{i}:(0, \infty) \times U\left(N_{i}\right) \rightarrow M$ is locally 1-1 near the regular point $(r, \xi)$. The assumption $\xi \in \mathcal{U}\left(N_{i}\right) \backslash \mathcal{U}_{i}$ implies the existence of a sequence of vectors $\xi_{n} \in U\left(N_{i}\right) \backslash\{\xi\}$ such that $\xi_{n} \rightarrow \xi$ as $n \rightarrow \infty$, and $c\left(\xi_{n}\right)=0$, i.e. $\rho\left(\Phi\left(t, \xi_{n}\right)\right)<t$ for all $t>0$. Let $x_{n}=\Phi\left(r, \xi_{n}\right)$; for each $n$, there exists $\xi_{n}^{\prime} \in \mathcal{U}(N)\left(\xi_{n}^{\prime} \neq \xi_{n}\right)$, and $r_{n}<r$ such that $x_{n}=\Phi\left(r_{n}, \xi_{n}^{\prime}\right)$. We claim that, for $n$ large, $\xi_{n}^{\prime} \notin \overline{\mathcal{U}\left(N_{i}\right)}$.In fact, assume that there exists a subsequence $\left\{\xi_{n_{k}}^{\prime}\right\} \subseteq \overline{\mathcal{U}\left(N_{i}\right)}$. It must accumulate to a vector $\xi^{\prime} \in \overline{\mathcal{U}\left(N_{i}\right)}$. Correspondingly, $r_{n_{k}}$ accumulates to a number $s \leq r$. Now since $x_{n} \rightarrow x$, we see that $\Phi\left(r_{n}, \xi_{n}^{\prime}\right) \rightarrow x$, so that $\Phi\left(s, \xi^{\prime}\right)=\Phi(r, \xi)$ with $s \leq r$. Since, by assumption, $r$ is the minimum distance of $x$ from $N$, we have necessarily $s=r$, i.e. $r_{n_{k}} \rightarrow r$. Now if $\xi^{\prime} \neq \xi$, we would have two distinct minimizing geodesics from $N$ to $x$, and this is impossible since otherwise the geodesic $t \rightarrow \Phi(t, \xi)$ would not minimize the distance past $r$. On the other hand, if $\xi^{\prime}=\xi$ both $\left(r_{n_{k}}, \xi_{n_{k}}^{\prime}\right)$ and $\left(r, \xi_{n_{k}}\right)$ converge to $(r, \xi)$, and this is incompatible with the fact that $\Phi$ is locally 1-1 near $(r, \xi)$, since $\Phi\left(r_{n_{k}}, \xi_{n_{k}}^{\prime}\right)=\Phi\left(r, \xi_{n_{k}}\right)$. The claim is then proved.

Hence, for $n$ large, $\xi_{n}^{\prime} \in \cup_{j \neq i} \overline{\mathcal{U}\left(N_{j}\right)}$, a compact set. Pick any accumulation point $\xi^{\prime}$ of $\left\{\xi_{n}^{\prime}\right\}$ and assume $\xi^{\prime} \in \overline{\mathcal{U}\left(N_{j}\right)}$. Reasoning as before, we see that $\xi^{\prime} \neq \xi$ is impossible, and so $\xi^{\prime}=\xi$, i.e. $\xi \in \overline{\mathcal{U}\left(N_{j}\right)} \subseteq \overline{U\left(N_{j}\right)}$, with $j \neq i$. If $\xi \in U\left(N_{j}\right)$, then $\pi(\xi) \in N_{j}$; but also $\pi(\xi) \in N_{i}$ and $j \neq i$ : impossible. Hence, necessarily $\xi \in \partial U\left(N_{j}\right)$.

The proofs of Propositions D.3 and D.4 are obvious adaptations of the proofs of Theorems 4.2 and 4.3 in [14].

Proposition D.3. - Let $\xi \in \mathcal{U}_{\mathrm{reg}}(N)$. If $\Phi(a, \xi)=\exp _{\pi(\xi)}$ a $\xi$ is the cut-point along the geodesic $t \rightarrow \Phi(t, \xi)$, then $\Phi(s, \xi) \in \mathcal{R}$ for all $0<s<a$. Moreover, we have one (or both) of the following alternatives:

(i) if $\xi \in \mathcal{U}_{i}$, then $\Phi(a, \xi)$ is the first focal point of $N_{i}$ along $t \rightarrow \Phi(t, \xi)$;

(ii) there are at least two minimizing geodesics from $N$ to $\Phi(a, \xi)$.

Proposition D.4. - Let $c: \mathcal{U}_{\mathrm{reg}}(N) \rightarrow[0, \infty]$ be the cut-radius map. Then $c$ is continuous.

Proposition D.5. - $M \backslash \mathcal{R}=\Phi(\operatorname{graph}(\mathrm{c})) \cup N \cup \Phi(\mathcal{F})$ with

$$
\mathcal{F}=\left\{(r, \xi) \in(0, \infty) \times \mathcal{U}_{\text {sing }}(N): 0<r \leq c(\xi)\right\}
$$

Proof. - Since $\Phi$ is surjective, if $x \in M \backslash \mathcal{R}$, and $x \notin N$, then $x=\Phi(r, \xi)$, for some $\xi \in \mathcal{U}(N), 0<r \leq c(\xi)$. If $\xi \in \mathcal{U}_{\text {sing }}(N)$, then $x \in \Phi(\mathcal{F})$. On the other hand, if $\xi \in \mathcal{U}_{\text {reg }}(N)$, then $r=c(\xi)$, otherwise $x \in \mathcal{R}$, by Proposition D.3. Hence in that case $x \in \Phi(\operatorname{graph}(c))$.

TOME $129-2001-\mathrm{N}^{\mathrm{O}} 4$ 
Proof of Theorem D.1. - Since $c: \mathcal{U}_{\text {reg }}(N) \rightarrow(0, \infty)$ is continuous, $\operatorname{graph}(c)$ has zero measure in $\mathcal{U}_{\text {reg }} \times(0, \infty)$ by Fubini's theorem, hence $\Phi(\operatorname{graph}(c))$ has zero measure in $M$; similarly, since $\mathcal{U}_{\text {sing }}(N)$ is contained in an $(n-2)$ - dimensional manifold, the set $\mathcal{F}$ is contained in an $(n-1)$-dimensional submanifold of $(0, \infty) \times U n(M)$ hence also $\Phi(\mathcal{F})$ has zero measure in $M$. Theorem D.1 then follows from Proposition D.5.

We let $\operatorname{Cut}(N)$ be the closure of $\Phi(\operatorname{graph}(c))$ in $M$. Then $\operatorname{Cut}(N)$ is a subset of $M \backslash \mathcal{R}$, and as such it has measure zero. As for $\Phi(\mathcal{F})$, this set consists of all points $\Phi(r, \xi), 0<r \leq c(\xi)$, with $\xi$ in the overlap of two different pieces $\overline{\mathcal{U}\left(N_{i}\right)}$ and $\overline{\mathcal{U}\left(N_{j}\right)}$ of the "unit normal bundle" $\mathcal{U}(N)$. If $c(\xi)<r$, then $\rho$ is $C^{1}$ at $\Phi(r, \xi)$, but not $C^{2}$. The reader is invited to draw a picture of the situation when $N$ is, for example, a triangle in the plane.

For a piecewise-smooth submanifold, integration in normal coordinates is the following formula:

$$
\int_{M} f=\sum_{i} \int_{\mathcal{R}_{i}} f=\sum_{i} \int_{\mathcal{U}_{i}} \int_{0}^{c(\xi)} f(\Phi(r, \xi)) \theta_{N_{i}}(r, \xi) \mathrm{d} r \mathrm{~d} \xi,
$$

and Lemma 2.1 becomes the following:

LEMma D.6. - Let $N=\cup_{i} N_{i}$ be a piecewise-smooth submanifold of $M$, and let $\rho$ be the distance function from $N$. Let $\Delta \rho$ be the distributional Laplacian of $\rho$. Then:

$$
\Delta \rho=\Delta_{\text {reg }} \rho+\Delta_{\text {Cut }} \rho-2 T
$$

where

$$
\left.\Delta_{\mathrm{reg}} \rho\right|_{\mathcal{R}_{i}} \circ \Phi_{i}=-\frac{\theta_{N_{i}}^{\prime}}{\theta_{N_{i}}}, \quad\langle T, \phi\rangle=\sum_{\left\{i: \operatorname{codim}\left(N_{i}\right)=1\right\}} \int_{N_{i}} \phi \mathrm{d} v_{n-1}
$$

for all $\phi \in C_{c}^{0}(M)$, and where $\Delta_{\text {Cut }} \rho$ is the positive measure defined by

$$
\left\langle\Delta_{\mathrm{Cut}} \rho, \phi\right\rangle=\sum_{i} \int_{\mathcal{U}_{i}} \theta_{N_{i}}(c(\xi), \xi) \cdot \phi\left(\exp _{\pi(\xi)} c(\xi) \xi\right) \mathrm{d} \xi .
$$

Proof. — Proceed as in the smooth case, with $N_{i}$ replacing $N$, and $\mathcal{U}_{i}$ replacing $U(N)$, and then sum over the index set $\mathcal{I}$. The proposition follows easily.

\section{BIBLIOGRAPHY}

[1] VAN DEN Berg (M.) \& GILKey (P.B.) - Heat content asymptotics for a Riemannian manifold with boundary, J. Funct. Anal., t. 120 (1994), pp. $48-71$.

[2] VAn Den Berg (M.) \& Le GALl (P.B.) - Mean curvature and the heat equation, Math. Z., t. 215 (1994), pp. 437-464.

BULletin DE LA SOCiÉtÉ MATHÉMATIQUE DE FRANCE 
[3] VAN DEN Berg (M.) \& SRisatKunARAJAh (S.) - Heat flow and brownian motion for a region in $\mathbb{R}^{2}$ with a polygonal boundary, Prob. Theory Rel. Fields, t. 86 (1990), pp. 41-52.

[4] Bishop (R.) \& Crittenden (R.) - Geometry of manifolds, Academic Press, New York, 1964.

[5] Burago (Yu. D.) \& Zalgaller (V. A.) - Geometric inequalities, A Series of Comprehensive Studies in Mathematics, vol. 285, Springer Verlag, 1988.

[6] Cheng (S.Y.) - Eigenvalue comparison theorems and its geometric applications, Math. Z., t. 142 (1975), pp. 289-297.

[7] Coddington (E. A.) - An Introduction to Ordinary Differential Equations, Prentice-Hall, Englewood Cliffs, N. J., 1961.

[8] Courtois (G.) - Comportement du spectre d'une variété riemannianne compacte sous perturbation topologique par excision d'un domaine, Ph.D. Thesis, Institut Fourier, Grenoble, 1987.

[9] Federer (H.) - Geometric measure theory, Springer Verlag, 1969.

[10] Gallot (S.) - Inégalités isopérimetriques et analitiques sur les variétés riemanniennes, Astérisque, t. 163-164 (1988), pp. 31-91.

[11] Gradshteyn (I.S.) \& Ryzhik (I.M.) - Table of integrals, series and products, Academic Press, 1980.

[12] Heintze (E.) \& KARCher (H.) - A general comparison theorem with applications to volume estimates for submanifolds, Ann. Sci. École Norm. Sup., t. 11 (1978), pp. 451-470.

[13] KAsue (A.) - On a lower bound for the first eigenvalue of the Laplace operator on a Riemannian manifold, Ann. Sci. École Norm. Sup., t. 17 (1984), no. 1, pp. 31-44.

[14] Kobayashi (S.) - On conjugate and cut-loci, Stud. Glob. Geom. and Analysis, Math. Ass. America (1967), pp. 96-122.

[15] Li (P.) \& YAU (S.-T.) - Estimates of eigenvalues of a compact Riemannian manifold, Proc. Symp. Pure Math., t. 36 (1980), pp. 205-239.

[16] SAVo (A.) - Une méthode de symétrization et quelques applications, C. R. Acad. Sci. Paris série I, t. 322 (1996), pp. 861-864.

[17] Uniform estimates and the whole asymptotic series of the heat content on manifolds, Geometriae Dedicata, t. 73 (1998), pp. 181-214.

[18] Simon (B.) - Functional integration and quantum physics, Academic Press, New York, 1980.

[19] Treves (F.) - Topological vector spaces, distributions and kernels, Academic Press, New York-London, 1962. 